\title{
Posterior Hippocampal Spindle Ripples Co-occur with Neocortical Theta Bursts and Downstates-Upstates, and Phase-Lock with Parietal Spindles during NREM Sleep in Humans
}

\author{
지 Jiang, ${ }^{1}$ Jorge Gonzalez-Martinez, ${ }^{3}$ and Eric Halgren ${ }^{1,2}$ \\ 'Department of Neurosciences and ${ }^{2}$ Department of Radiology, University of California at San Diego, La Jolla, California 92093, and ${ }^{3}$ Epilepsy Center, \\ Cleveland Clinic, Cleveland, Ohio 44106
}

\begin{abstract}
Human anterior and posterior hippocampus $(\mathrm{aHC}, \mathrm{pHC})$ differ in connectivity and behavioral correlates. Here we report physiological differences in humans of both sexes. During NREM sleep, the human hippocampus generates sharpwave ripples (SWRs) similar to those which in rodents mark memory replay. We show that while $\mathrm{pHC}$ generates SWRs, it also generates approximately as many spindle ripples (SSR: ripples phase-locked to local spindles). In contrast, SSRs are rare in aHC. Like SWRs, SSRs often co-occur with neocortical theta bursts (TBs), downstates (DSs), sleep spindles (SSs), and upstates (USs), which coordinate cortico-hippocampal interactions and facilitate consolidation in rodents. SWRs co-occur with these waves in widespread cortical areas, especially frontocentral. These waves typically occur in the sequence TB-DS-SS-US, with SWRs usually occurring before SS-US. In contrast, SSRs occur $\sim 350 \mathrm{~ms}$ later, with a strong preference for co-occurrence with posterior-parietal SSs. pHC-SSs were strongly phase-locked with parietal-SSs, and pHC-SSRs were phase-coupled with pHC-SSs and parietal-SSs. Human SWRs (and associated replay events, if any) are separated by $\sim 5 \mathrm{~s}$ on average, whereas ripples on successive SSR peaks are separated by only $\sim 80 \mathrm{~ms}$. These distinctive physiological properties of pHC-SSR enable an alternative mechanism for hippocampal engagement with neocortex.
\end{abstract}

Key words: electrophysiology; hippocampus; human; memory; neocortex; sleep

\section{Significance Statement}

Rodent hippocampal neurons replay waking events during sharpwave ripples (SWRs) in NREM sleep, facilitating memory transfer to a permanent cortical store. We show that human anterior hippocampus also produces SWRs, but spindle ripples predominate in posterior. Whereas SWRs typically occur as cortex emerges from inactivity, spindle ripples typically occur at peak cortical activity. Furthermore, posterior hippocampal spindle ripples are tightly coupled to posterior parietal locations activated by conscious recollection. Finally, multiple spindle ripples can recur within a second, whereas SWRs are separated by $\sim 5 \mathrm{~s}$. The human posterior hippocampus is considered homologous to rodent dorsal hippocampus, which is thought to be specialized for consolidation of specific memory details. We speculate that these distinct physiological characteristics of posterior hippocampal spindle ripples may support a related function in humans.

\section{Introduction}

Hippocampal sharpwave ripples (HC-SWRs) are striking complexes of local field potentials (LFPs) recorded during NREM sleep and rest periods (Buzsáki, 2015). Pyramidal cell firing during HC-SWR tends to reproduce the spatiotemporal patterns established during the preceding active periods; this "replay" is
Received Nov. 6, 2018; revised June 26, 2019; accepted July 13, 2019.

Author contributions: X.J. and E.H. designed research; X.J. performed research; X.J. analyzed data; X.J. and E.H. wrote the first draft of the paper; X.J. and E.H. edited the paper; X.J. and E.H. wrote the paper; J.G.-M. contributed unpublished reagents/analytic tools.

This work was supported by the U.S. Office of Naval Research's Multidisciplinary University Research Initiatives Program N00014-16-1-2829, National Institute of Mental Health RF1 MH117155 and R01 MH111437, and National Institute of Biomedical Imaging and Bioengineering R01 EB009282. We thank John Gale, Qianqian Deng,
Charles Dickey, Darlene Evardone, Zach Fitzgerald, Chris Gonzalez, Don Hagler, Milan Halgren, Erik Kaestner, Rachel Mak-McCully, Adam Niese, Burke Rosen, and T. G. Venti for support.

The authors declare no competing financial interests.

Extended Data is available at https://www.jneurosci.org/content/39/45/8949/tab-figures-data\#fig-datasupplementary-materials.

Correspondence should be addressed to Xi Jiang at x4jiang@ucsd.edu or Eric Halgren at ehalgren@ucsd.edu.

https://doi.org/10.1523/JNEUROSCI.2858-18.2019

Copyright $\odot 2019$ the authors 
hypothesized to guide cortical activity as it consolidates memories (Wilson and McNaughton, 1994; Skaggs and McNaughton, 1996; Nádasdy et al., 1999). Disrupting HC-SWR impairs memory consolidation (Girardeau et al., 2009; Maingret et al., 2016). The coordination of $\mathrm{HC}$ ripples with cortical consolidation is thought to be orchestrated by neocortical LFP graphoelements (NC-GEs) such as sleep spindles (SSs), downstates (DSs), and upstates (USs), and possibly theta bursts (TBs) (Gonzalez et al., 2018). Specifically, in rodents, evidence suggests that coordination between HC-SWRs and NC-SSs (Siapas and Wilson, 1998; Sirota et al., 2003; Peyrache et al., 2009) and DSs/USs (Battaglia et al., 2004; Mölle et al., 2006; Headley et al., 2017) is central to replay (Diekelmann and Born, 2010; O’Neill et al., 2010; Girardeau and Zugaro, 2011), and recent human data also point to NC-TB, NC-SS, and NC-US coordination being involved in targeted memory reactivation (Göldi et al., 2019). The NC-GEs themselves have systematic temporal relations, typically occurring in the order TBs $\rightarrow$ DSs $\rightarrow$ SSs $\rightarrow$ USs (Mak-McCully et al., 2017; Gonzalez et al., 2018).

HC-SWRs have been extensively studied in rodents. However, two macaque studies have confirmed the presence of HC-SWRs during NREM sleep with the typical biphasic negative-positive waves in radiatum, and $\sim 100 \mathrm{~Hz}$ ripples in pyramidale (Skaggs et al., 2007; Ramirez-Villegas et al., 2015). HC-SWRs have also been observed in recordings from hippocampal electrodes implanted in humans to identify epileptogenic tissue. In a series of studies, Bragin et al. (1997, 1999) described HC-SWRs but mainly focused on the use of ripples to identify epileptogenic tissue. Axmacher et al. (2008) described HC ripples that occurred mainly in waking but were phase-locked to $\delta$. Previously, we described typical SWRs in human HC (Jiang et al., 2019).

Recently, Staresina et al. (2015) reported that HC ripples in humans during NREM are tightly phase-coupled to hippocampal SSs (HC-SSs) while presenting no clear evidence for sharp-wave ripples. In this study, we aimed to confirm the presence of ripples associated with HC-SSs (henceforth referred to as HC-SSRs), and to compare their characteristics and NC-GE associations (if any) with those of HC-SWRs, which we reported previously (Jiang et al., 2019). Furthermore, since Staresina et al. (2015) recorded HC-SSRs mainly in posterior HC ( $\mathrm{pHC})$, whereas previous human $\mathrm{HC}$ recordings were mainly in anterior $\mathrm{HC}(\mathrm{aHC})$ where most of the human hippocampal volume lies (Destrieux et al., 2013), we examined whether HC-SSRs selectively occurred in pHC, with HC-SWRs dominant in aHC. The possibility that aHC versus $\mathrm{pHC}$ have distinct LFP contexts is also suggested by their distinct anatomy and functional correlates (Ranganath and Ritchey, 2012; Poppenk et al., 2013; Strange et al., 2014). Furthermore, since cortical SSs preferentially occur in NREM Stage N2 compared with N3 (Mak-McCully et al., 2017), we contrasted these stages when performing our NC-GE to HC-GE association analyses. Finally, provided that HC-SSRs can be identified, we sought to determine their temporal relationship to HC-SWRs, as well as NC-GEs.

We report here that, indeed, both SWRs and SSRs exist in the $\mathrm{HC}$, but with distinct anatomy, cortical relations, and timing. Whereas HC-SSRs and HC-SSs are largely confined to pHC, HCSWRs predominate in aHC. pHC-SSs and pHC-SSRs tend to co-occur with NC-SSs, especially in parietal sites, whereas aHCSWRs co-occur with more extensive cortical locations, especially frontal. While aHC-SWRs usually occur before SSs/USs, pHCSSs/SSRs tend to occur during NC-SSs/USs, following NCTBs/DSs. Hippocampal and parietal spindles become highly synchronous in $\sim 2$ cycles, and both modulate HC ripples. Thus,
pHC-SSRs interact in close synchrony with parietal NC-SSs within the context of more widespread cortical rhythms during NREM. The contrasting anatomy and physiology of pHC-SSRs and aHC-SWRs provide another possible mechanism for hippocampocortical coordination during NREM sleep.

\section{Materials and Methods}

Patient selection. Twenty patients with long-standing drug-resistant partial seizures underwent stereoelectroencephalography (SEEG) depth electrode implantation to localize seizure onset and thus direct surgical treatment (for demographic and clinical information, see Table 1). Patients were selected from a group of 54 for minimal pathology. The 20 remaining patients included 7 males, $29.8 \pm 11.9$ years of age (range $16-58$ years). Electrode targets and implantation durations were chosen entirely on clinical grounds (Gonzalez-Martinez et al., 2013). All patients gave fully informed consent for data usage as monitored by the local Institutional Review Board, in accordance with clinical guidelines and regulations at Cleveland Clinic.

Electrode localization. After implantation, electrodes were located by aligning postimplant CT to preoperative 3D T1-weighted structural MRI with $\sim 1 \mathrm{~mm}^{3}$ voxel size (Dykstra et al., 2012), using 3D Slicer (RRID: SCR_005619). This allows visualization of individual contacts with respect to HC cross-sectional anatomy, which was interpreted in reference to the atlas of Duvernoy (1988). The assignment of depth contacts to $\mathrm{aHC} / \mathrm{pHC}$ was made with the posterior limit of the uncal head as boundary (Poppenk et al., 2013; Ding and Van Hoesen, 2015). Recordings were obtained from $32 \mathrm{HC}$ contacts: 20 anterior (11 left) and 12 posterior (7 left). In 4 patients, $\mathrm{HC}$ recordings were bilateral ( 3 anterior and 1 posterior); and in 8 patients, ipsilateral $\mathrm{aHC} / \mathrm{pHC}$ were both recorded. The distance of each hippocampal contact from the anterior limit of the hippocampal head was obtained in Freesurfer (RRID:SCR_001847). The CT-visible cortical contacts were then identified as previously described (Jiang et al., 2019), to ensure that activity recorded by bipolar transcortical pairs is locally generated (Mak-McCully et al., 2015). Electrode contacts were rejected from analysis if they were involved in the early stages of the seizure discharge or had frequent interictal activity or abnormal spontaneous LFPs. From the total of 2844 contacts implanted in the 20 patients, 366 transcortical pairs (18.3 \pm 4.7 per patient) were accepted for further analysis. Polarity of the pairs was adjusted, if necessary, to "pial surface minus white matter" according to MRI localization, confirmed with decreased high $\gamma$ power during surface-negative DSs (see below).

Freesurfer (Dale et al., 1999; Fischl et al., 1999a, 2004) was used to reconstruct from individual MRI scans the cortical pial and inflated surfaces, as well as automatic parcellation of the cortical surface into anatomical areas (Desikan et al., 2006), after a sulcal-gyral alignment process. An average surface for all 20 patients was then generated to serve as the basis of all 3D maps. While each cortical SEEG electrode contact's location was obtained through direction correlation of CT and MRI as described earlier in this section, we obtained the cortical parcellation labels corresponding to each contact by morphing the right-anteriorsuperior-oriented anatomical coordinates from individual surfaces to the average surface space (Fischl et al., 1999b). In addition, the nearest vertices on the average surface to the morphed coordinates would be identified for subsequent plotting of 2D projections onto the left lateral view (see Fig. 4). For the 2D projections only, to optimize visualization (i.e., minimize multiple contact overlap) while preserving anatomical fidelity, positions of contacts with significant HC-NC GE correlation were allowed to shift within a $5 \mathrm{~mm}$ radius. All visualizations were created with custom scripts in MATLAB 2016b (The MathWorks).

Data collection and preprocessing. Continuous recordings from SEEG depth electrodes were made with cable telemetry system (JE-120 amplifier with 128 or 256 channels, $0.016-3000 \mathrm{~Hz}$ bandpass, Neurofax EEG1200, Nihon Kohden) across multiple nights (Tables 1, 2) over the course of clinical monitoring for spontaneous seizures, with $1000 \mathrm{~Hz}$ sampling rate. The total NREM sleep durations vary across patients; while some difference is expected given intrinsic variability of normal human sleep duration (Carskadon and Dement, 2010) and sleep deprivation in clini- 
Table 1. List of patients, age, sex, handedness, language dominance, NC channel counts, and the lengths sleep period recordings used ${ }^{a}$

\begin{tabular}{|c|c|c|c|c|c|c|c|c|c|c|c|}
\hline $\begin{array}{l}\text { Patient } \\
\text { no. }\end{array}$ & $\begin{array}{l}\text { Age } \\
\text { (yr) }\end{array}$ & Sex & Handedness & $\begin{array}{l}\text { No. of } \\
\text { cortical } \\
\text { channels }\end{array}$ & $\begin{array}{l}\mathrm{HC} \\
\text { seizure-free? }\end{array}$ & $\begin{array}{l}\mathrm{HC} \\
\text { interictal-free? }\end{array}$ & $\begin{array}{l}\text { No. of } \\
\text { sleep } \\
\text { periods }\end{array}$ & $\begin{array}{l}\text { Mean } \\
\text { duration } \\
\text { of NREM (h) }\end{array}$ & $\begin{array}{l}\text { SD } \\
\text { NREM (h) }\end{array}$ & $\begin{array}{l}\text { Total N2 } \\
\text { duration (h) }\end{array}$ & $\begin{array}{l}\text { Total N3 } \\
\text { duration (h) }\end{array}$ \\
\hline 1 & 20 & $M$ & $\mathrm{R}$ & 19 & $Y$ & $\mathrm{~N}$ & 3 & 2.2 & 0.78 & 2.8 & 3.3 \\
\hline 2 & 51 & $\mathrm{~F}$ & $\mathrm{R}$ & 12 & $Y$ & $\mathrm{~N}$ & 4 & 7.5 & 1.87 & 27.3 & 0 \\
\hline 3 & 58 & $\mathrm{~F}$ & $\mathrm{R}$ & 24 & $Y$ & $\mathrm{~N}$ & 4 & 7.1 & 2.44 & 23.1 & 0.30 \\
\hline 4 & 42 & $M$ & $\mathrm{~L}$ & 17 & $Y$ & $\mathrm{~N}$ & 4 & 3.1 & 0.33 & 1.8 & 9.0 \\
\hline 5 & 18 & $\mathrm{~F}$ & $\mathrm{~L}$ & 21 & $Y$ & $Y$ & 1 & 3.7 & & 1.9 & 0.9 \\
\hline 6 & 20 & $\mathrm{~F}$ & $\mathrm{R}$ & 21 & $Y$ & $\mathrm{~N}$ & 3 & 2.7 & 1.49 & 2.6 & 3.0 \\
\hline 7 & 22 & $M$ & $\mathrm{LR}$ & 19 & $Y$ & $Y$ & 3 & 3.8 & 1.17 & 2.4 & 3.8 \\
\hline 8 & 30 & $\mathrm{~F}$ & $\mathrm{R}$ & 13 & $Y$ & $\mathrm{~N}$ & 5 & 5.2 & 1.26 & 6.5 & 14 \\
\hline 9 & 43 & $\mathrm{~F}$ & $\mathrm{R}$ & 12 & $Y$ & $\mathrm{~N}$ & 4 & 3.1 & 0.45 & 3.7 & 4.4 \\
\hline 10 & 16 & $M$ & $\mathrm{R}$ & 17 & Y & $Y$ & 5 & 3.8 & 1.11 & 7.5 & 8.8 \\
\hline 11 & 32 & $\mathrm{~F}$ & $\mathrm{R}$ & 30 & $Y$ & $\mathrm{~N}$ & 3 & 5.1 & 3.43 & 8.4 & 2.8 \\
\hline 12 & 36 & M & $\mathrm{L}$ & 25 & $Y$ & $Y$ & 4 & 5.2 & 1.48 & 11.6 & 6.5 \\
\hline 13 & 21 & $F$ & $\mathrm{~L}$ & 14 & $Y$ & $\mathrm{~N}$ & 3 & 6 & 0.47 & 14.7 & 1.1 \\
\hline 14 & 21 & $\mathrm{~F}$ & $\mathrm{R}$ & 14 & $Y$ & $\mathrm{~N}$ & 8 & 3.7 & 1.1 & 16.9 & 9.3 \\
\hline 15 & 29 & $\mathrm{~F}$ & $\mathrm{R}$ & 17 & $Y$ & $\mathrm{~N}$ & 4 & 2.5 & 1.31 & 5.4 & 2.7 \\
\hline 16 & 41 & $\mathrm{~F}$ & $\mathrm{R}$ & 19 & $Y$ & $\mathrm{~N}$ & 3 & 4.6 & 0.72 & 7.4 & 4.4 \\
\hline 17 & 24 & $M$ & $\mathrm{R}$ & 21 & $Y$ & $\mathrm{~N}$ & 3 & 5.1 & 1.88 & 8.9 & 2.9 \\
\hline 18 & 31 & $\mathrm{~F}$ & $\mathrm{R}$ & 15 & $Y$ & $\mathrm{~N}$ & 6 & 6.1 & 1.18 & 24.2 & 3.8 \\
\hline 19 & 21 & $M$ & $\mathrm{R}$ & 15 & $Y$ & $Y$ & 4 & 2.8 & 0.72 & 5.4 & 5.8 \\
\hline 20 & 19 & $\mathrm{~F}$ & $\mathrm{R}$ & 21 & $Y$ & $Y$ & 3 & 4.9 & 2.53 & 5.2 & 6.0 \\
\hline Mean & 30 & & & 18 & $\mathrm{~T}: 20$ & $\mathrm{~T}: 8$ & 4 & 4.4 & & 9.39 & 4.64 \\
\hline
\end{tabular}

a Reproduced with permission from Jiang et al. (2019).

cal environment, we also confirmed that the percentages of NREM in total sleep from 28 sleeps across 16 of our patients were comparable to (i.e., within 2 SD of) normative data (Moraes et al., 2014) in terms of N2 and N3 durations, with no significant difference in GE occurrence rates between normative and other sleep (Jiang et al., 2019). Recordings were anonymized and converted into the European Data Format. Subsequent data preprocessing was performed in MATLAB (RRID:SCR_001622); the Fieldtrip toolbox (Oostenveld et al., 2011) was used for bandpass filters, line noise removal, and visual inspection. Separation of patient NREM sleep/wake states from intracranial LFP alone was achieved by previously described methods using clustering of first principal components of $\delta$-to-spindle and $\delta$-to- $\gamma$ power ratios across multiple LFPderived signal vectors (Gervasoni et al., 2004; Jiang et al., 2017), with the addition that separation of $\mathrm{N} 2$ and $\mathrm{N} 3$ was empirically determined by the proportion of DSs that are also part of slow oscillations (at least $50 \%$ for N3) (Silber et al., 2007), since isolated DSs in the form of K-complexes are predominantly found in Stage 2 sleep (Cash et al., 2009).

Hippocampal GE selection. To identify hippocampal spindles, we applied a previously reported spindle detector (Mak-McCully et al., 2017) to hippocampal LFP signals from NREM: a $10-16 \mathrm{~Hz}$ bandpass (zerophase shift filter with transition bands equal to $30 \%$ of the cutoff frequencies) was applied to the LFP data, and the analytic amplitude of the bandpassed signal was convolved with an average Tukey window of 600 $\mathrm{ms}$. A channel-wise cutoff set at mean $\pm 2 \mathrm{SD}$ was applied to identify local maxima that would be "peaks" of putative spindles, with spindle edges defined at $50 \%$ of peak amplitude. The putative HC-SS segments were accepted for subsequent analyses if they were $>400 \mathrm{~ms}$, had $<14 \mathrm{~dB}$ power in the nearby frequency bands $(4-8 \mathrm{~Hz}$ and $18-30 \mathrm{~Hz})$, and contained at least 3 unique oscillation peaks with 40-100 ms gaps.

Hippocampal SWRs were identified by starting with the top $20 \%$ of peaks in root-mean-square amplitude of $60-120 \mathrm{~Hz}$ LFP over a moving $20 \mathrm{~ms}$ window, then requiring that putative SWR events: (1) be $>200 \mathrm{~ms}$ apart; (2) have $>3$ distinct ripple peaks in the HC LFP signal (low-passed at $120 \mathrm{~Hz}$ ); and (3) be associated with an LFP matching hand-marked sharp-waves in the same subject and channel (Jiang et al., 2019). RMS peaks potentially arising from artifacts or epileptiform activity were rejected by detecting sharp LFP transients and setting a threshold that separated hand-marked HC-SWR from interictal events (Jiang et al., 2019). Before the SWR similarity evaluation, putative ripples were also examined for whether a given ripple center falls within a hippocampal spindle duration in the same signal; if positive, such ripples were then deemed SSR. Since a single ripple could qualify as both SWR and SSR, such a ripple would be denoted as SXR where appropriate.

Cortical GE selection. Automatic cortical TB detection was performed as previously described (Gonzalez et al., 2018): a 5-9 Hz zero-phase shift bandpass filter was applied to the LFP data, and a channel-wise cutoff set at mean $\pm 3 \mathrm{SD}$ was applied to each channel's Hilbert envelope (smoothed with $300 \mathrm{~ms}$ Gaussian kernel) to identify local maxima that would be "peaks" of putative TBs, and make start/stop edge cutoffs at mean \pm SD. The resulting TBs were only accepted for further analyses if their durations fall between 400 and $1000 \mathrm{~ms}$, with their number-ofzero-crossings-based frequency estimate falling within the bandpass frequency range as well.

Automatic cortical spindle (SS) detection was performed in the same matter as previously described for hippocampal spindles. DSs and USs were identified as follows: for each cortical LFP signal in NREM, a zerophase filter from 0.1 to $4 \mathrm{~Hz}$ was applied. Consecutive zero crossings of opposite slope separated by $250-3000 \mathrm{~ms}$ were then selected as delineating putative GEs. For each putative GE, the amplitude peak between zero crossing was computed; only the top $10 \%$ of peaks were retained. The polarity of each signal was inverted, if necessary, to assure that DSs were negative, as confirmed with a high $\gamma(70-190 \mathrm{~Hz})$ power decrease exceeding $1 \mathrm{~dB}$ within $\pm 250 \mathrm{~ms}$ of the negative DS peaks.

Experimental design and statistical analysis. Statistical tests that involve multiple comparisons in this and subsequent Materials and Methods subsections all have $\alpha=0.05$ after FDR correction (Benjamini and Hochberg, 1995). The FDR correction procedure was implemented as follows: for a total of $N$ hypotheses, each with corresponding $p$ values $P_{i}(i \in N)$, which are sorted in ascending order to identify $P_{k}, k$ being the largest $i$ for which $P_{i} \leq$ $(i / N) \times \alpha$. All hypothesis with $p$ values $\leq P_{k}$ would then be rejected. The striking positive skewness both in general and in specific categories of the NC-GE histograms (Extended Data Fig. 3-1) suggests high variability in co-occurrence rates of NC-GEs with HC-SSs and with HC-SSRs. Therefore, to account for sampling error, we created linear mixed-effect (LME) models for several analyses as described below.

We compared the occurrence rates of HC-SSRs/SSs from different NREM stages (N2 vs N3) and from different sources (aHC vs pHC) with both paired $t$ tests and LME models to account for patient-wise variability. For LME, our base models have either HC-SSR or HC-SS rates as the response variable, with $\mathrm{AP}(\mathrm{aHC} / \mathrm{pHC})$ and stage $(\mathrm{N} 2 / \mathrm{N} 3)$ as categorical predictor variables. The full model includes interaction between predictor variables and all possible random slope/intercept terms (GE rate $\sim 1+\mathrm{AP} \times$ 
stage $+($ AP $\mid$ patient ID $)+($ stage $\mid$ patient ID $)+(1 \mid$ patient ID $))$, with reduced models' fits being compared with the full model fit via likelihood ratio tests. The best-fit models for both HC-SSRs and HC-SSs turned out to be the following: $\mathrm{HC}-\mathrm{GE}$ rate $\sim 1+\mathrm{AP} \times$ stage $+(\mathrm{AP} \mid$ patient $\mathrm{ID})+(1 \mid$ patient ID).

Similarly, we compared the occurrence rates of HC-SWRs with those of HC-SSRs in different NREM stages and from different hippocampal origins, while accounting for random effect due to patient identity. Our full LME model has HC-GE rates as the response variable, with three categorical predictor variables and all possible random slopes, plus random intercept due to patient identity. By stepwise removal of individual terms from the full model and comparing each reduced model with the one before it via likelihood ratio tests, we arrived at the best fit model of GE rate $\sim 1+$ GE type $\times$ AP $\times$ stage $+($ GE type $\mid$ patient ID).

We also evaluated whether a significant difference between SSR/SWR ratio in the first versus second half of NREM sleep could be found while accounting for variance due to patient identity. Our base LME model has SSR/SWR ratio as the response variable, and "sleep halves" as the categorical predictor variable. After comparing models with random intercept and/or slope with base model, we found the best fit to be the base model (ratio $\sim 1+$ sleep halves).

For time-frequency analysis in Figure 1, spectral content of the LFP data from hippocampal channels was evaluated using EEGLAB (RRID: SCR_007292) routines that applied wavelet transforms (Delorme and Makeig, 2004). Spectral power was calculated over 1-200 Hz across each individual time period ("trial") centered around HC-SWR ripple centers, HC-SSR ripple centers, or HC-SS onsets found in NREM by convoluting each trial's signal with complex Morlet wavelets, and averages were then taken across trials. The resulting time-frequency matrices were normalized with respect to the mean power at each chosen frequency and masked with two-tailed bootstrap significance at $\alpha=0.05$, with the pretrigger times $(-2000 \mathrm{~ms}$ to $-1500 \mathrm{~ms}$ ) as baseline.

Peristimulus time histograms were constructed for each HC-NC channel pair, separately for each GE (TB, SS, DS, and US), and for each sleep-stage (N2 or N3), each histogram comprising the occurrence times of a given NC-GE during the $\pm 2 \mathrm{~s}$ interval surrounding midpoints of HC-SSRs and onsets of HC-SSs (for examples, see Fig. 3). Together, there were 598 unique HC-NC pairs (458 ipsilateral, 140 contralateral), and 16 histograms for each pair (one for each sleep stage, times each of the 4 GE types, for HC-SSs or SSRs), for a total of 9568 histograms. On average, each of these histograms plots the occurrence times of $1386 \pm 2565$ NC-GEs (3.86 skewness) with respect to $861 \pm 1581$ HC-SSRs (3.57 skewness) and to $1022 \pm 1642$ HC-SSs (3.61 skewness). Overall, a total of 13.2 million NC-GE events, 4.12 million HC-SSRs, and 4.89 million HC-SSs were plotted.

The significance of peaks and troughs in each histogram was tested by comparing them with distributions derived from histograms constructed under the null hypothesis of no relationship between the NC-GEs and HC-SS/SSRs using the following randomization procedure. Nullhypothesis histograms $(N=1000)$ were constructed of NC-GE occurrences relative to a number of random times equal to the number of HC-SSs/SSRs (for examples, see Fig. 3). For each $200 \mathrm{~ms}$ time bin with $100 \mathrm{~ms}$ overlap comprising the $4 \mathrm{~s}$ trial duration, the actual counts are then compared with the distribution under the null hypothesis, followed by FDR correction. Similar peristimulus histograms and randomization tests were also constructed with aHC-SWRs as triggers and pHC-SWRs/ SSRs as tallied events (see Fig. 2A).

The latencies of the largest significant peaks, identified in the individual histograms constructed as described above, were used to create summary histograms for each GE, sleep stage, and HC-SS/SSR origin in aHC versus pHC. These are plotted in Figure 4 and tabulated in Table 3. Each of the 16 histograms of histogram peaks $(\mathrm{N} 2 / \mathrm{N} 3 \times \mathrm{aHC} / \mathrm{pHC} \times 4 \mathrm{NC}-$ GEs) for HC-SSRs summarizes the significant latencies from $78 \pm 41$ HC-NC channel pair histograms, comprising a total of $1.37 \times 10^{5} \pm$ $1.45 \times 10^{5} \mathrm{HC}$-NC events (range $7.18 \times 10^{3}$ to $4.04 \times 10^{5}$ ). Each of the 16 histograms of histogram peaks for HC-SSs summarizes the significant latencies from $126 \pm 63 \mathrm{HC}-\mathrm{NC}$ channel pair histograms, comprising a total of $2.46 \times 10^{5} \pm 2.07 \times 10^{5} \mathrm{HC}-\mathrm{NC}$ events (range $1.24 \times 10^{4}$ to $\left.6.23 \times 10^{5}\right)$. To test whether, overall, a given type of cortical GE signifi- cantly precedes or follows HC-SSs/SSRs, two-tailed binomial tests with chance probability of 0.5 were performed on the number of channel pairs with peak latencies in the $2000 \mathrm{~ms}$ before versus the $2000 \mathrm{~ms}$ after the reference HC-SSs/SSRs. Since certain GEs, such as cortical $\theta$, tend to yield peak latencies centered around zero, we also tested with KolmogorovSmirnov tests whether, overall, for a given NC-GE, the distribution of peak latencies significantly related to HC-SSs/SSRs differs from chance.

To evaluate the phase relationship between cortical and hippocampal spindle oscillations, we computed phase-locking values (PLVs) (Lachaux et al., 1999) between each cortical and hippocampal channel pairing over $3 \mathrm{~s}$ trials centered on all hippocampal spindle event starts in NREM $(\mathrm{N} 2+\mathrm{N} 3)$. Only hippocampal GEs that overlapped with the cortical spindle for at least $160 \mathrm{~ms}$ were included, to ensure that the cortical and hippocampal spindles overlap for at least 1 spindle cycle, thus allowing more accurate estimates of phase-locking. We also computed PLV for the same cortical-hippocampal channel pairs over the same number of trials centered on random times in NREM to create a baseline estimate; and for each nonoverlapping $50 \mathrm{~ms}$ time bin, a two-sample $t$ test was performed between the actual PLV and the baseline estimate, with the resulting $p$ values undergoing FDR correction. A given channel pair would be considered significantly phase-locking if: (1) >40 trials were used in the PLV computation, since small sample size is known to introduce bias (Aydore et al., 2013); or (2) at least 3 consecutive time bins yield post-FDR $p$ values $<0.05$.

To evaluate phase-amplitude coupling (PAC) between cortical spindle phase and hippocampal ripple amplitude, we adopted a Modulation Index method as follows (Tort et al., 2010): for each HC-NC channel pair with significant SS PLV, we first computed instantaneous phase and amplitude through Hilbert transform for each trial (as previously obtained for PLV analysis), with NC-SS phase angles in 10 degree bins to create a corresponding mean amplitude distribution across trials. For statistical evaluation of observed PAC, we then computed the Modulation Index (i.e., dividing the Kullback-Leibler distance of the observed amplitude distribution from the uniform distribution by $\log (N), N$ being the number of trials) for this channel pair, and performed permutation tests by generating 200 additional Modulation Indices with random pairings of the NC-SS phase and HC ripple amplitude (so that phase from one trial is matched to amplitude from another trial). The permutation test results were then FDR-corrected.

For intrahippocampal coupling between ripple-frequency amplitude $(70-100 \mathrm{~Hz})$ and spindle $(10-16 \mathrm{~Hz})$ phase, we applied previously published methods for evaluating event-related PAC (ERPAC) and estimating subsequently ERPAC significance over time from intracranial LFP data (Voytek et al., 2013). The events were NREM hippocampal spindle starts, and each ERPAC trial covers -1000 to $1500 \mathrm{~ms}$ perievent. Each trial was also marked as either overlapping with NC-SS (by more than one spindle cycle) or not. Statistical evaluation of significant coupling was done using permutation tests that kept the actual analytic amplitude and phase values at each time point, but randomized the trial labels 1000 times so that the amplitude values are matched to phase values from random trials. The resulting $p$ values for each time point then underwent FDR correction. To generate the comodulogram in Figure 2C, we concatenated the hippocampal data across spindle events and applied previously published PAC algorithm (Colgin et al., 2009) implemented within the Pactools software package (Dupréla Tour et al., 2017) under Python 3.6 (RRID:SCR_008394).

To explore the anatomical distribution of HC-NC GE co-occurrences, we tested the following: (1) whether the distribution of significantly SSRor HC SS-coupled NC sites was different from chance for a given HC site; (2) whether the significantly different from chance distributions of NC sites from 1 would also differ between aHC/pHC site pairs; and (3) whether the NC sites showing significant $\mathrm{HC}-\mathrm{NC}$ correlation with one NC-GE type tended to also show correlation with another NC-GE type.

For 1 , we performed a $\chi^{2}$ test of homogeneity for each $\mathrm{HC}$ site with regard to each GE, that is, for a given patient with multiple NC sites, a $2 \times$ $N$ contingency table was made for each $\mathrm{NC}$ site, $N$ being the number of $\mathrm{NC}$ sites, one row of table containing the number of NC-GE overlapping with (i.e., occurring within $1500 \mathrm{~ms}$ of) HC-SS/SSR for each NC, the other containing the number of NC-GE that do not. A significant $\chi^{2}$ would therefore indicate a significant nonrandom distribution for that particular $\mathrm{HC}$ site and NC-GE type. 
For 2, we performed Wilcoxon signed rank tests on the data obtained in 1 to evaluate whether for a given NC-GE type (for which 1 yielded significance for both members of an HC site pair), the proportions of GE from significantly coupled NC sites that overlapped with aHC-SSRs/SSs differ from the proportions for $\mathrm{pHC}$-SSR/SS. Each test required that both members of a HC site pair have a significant $\chi^{2}$ test result from 1 .

We further explored the spatial distribution of HC-NC relationship by tallying the proportion of significant HC-NC channel pairs across different NC regions, with respect to different NC-GE types and different HC-SS/SSR sources (Table 4, Table 5). To characterize the apparent variability in Tables 4 and 5, we performed four-way ANOVAs to compare the main effects of GE type (TB, SS, DS, US), NREM stage (N2 vs N3), aHC or pHC origin of HC-SS/SSR, and NC ROIs (coverage listed in Extended Data Table 4-1) as well as the two-way and three-way interaction effects (Table 6, Table 7). To evaluate the differences in NC-HC coupling between SWRs and SSRs, we combined the SSR data with data from the equivalent analysis in our study on SWR (Jiang et al., 2019), and performed another four-way ANOVA with the following factors: SSR/SWR as the participant of NC-HC coupling, NREM stage, aHC/pHC origin of HC-SWR/SSR, and NC ROIs.

Similarly, given the lateralization of human hippocampal function, we were interested in examining whether ipsilateral HC-NC channel pairs would show different relationships from contralateral pairs across GE types, NREM stages, or NC ROIs. Due to a sparse representation of contralateral channel pairs for individual ROIs, we calculated proportions for larger regions by combining both $\mathrm{HC}$ and $\mathrm{NC}$ sites: $\mathrm{aHC}$ and pHC channels were combined into a single HC category, and the $10 \mathrm{NC}$ ROIs used in previous analysis were collapsed into two: "frontocentral" and "nonfrontal" (Extended Data Table 4-1). We then computed for each ROI the proportion of NC channels significantly coupled to HCSSs/SSRs (Table 8), and performed four-way ANOVA as previously described, with the ipsilateral/contralateral factor replacing the aHC/pHC factor. All post hoc analyses for both ANOVAs were performed with Tukey's range test. For each ANOVA, we checked the normality assumption by conducting the Lilliefors test on the residuals.

Code accessibility. All custom scripts would be available upon request by contacting the corresponding author and would be delivered through the UCSD RDL-share service.

\section{Results}

In this paper, we quantified HC-SSs and HC-SSRs in humans, their relationship to $\mathrm{HC}$-SWRs and NC-GEs, and their variation within different $\mathrm{HC}$ regions and sleep stages. We confirmed that ripples phase-modulated by local SSs (i.e., HC-SSRs) do occur in the human hippocampus, but they are preferentially located in the pHC sites. Like HC-SWRs (Jiang et al., 2019), HC-SS/SSRs were strongly related to the NC-GEs in a spatiotemporally diverse manner, with a particularly strong relationship in Stage N2 between pHC-SSs/SSRs and NC-TBs, DSs, SSs, and USs. The relative latencies are consistent with the order we have established in earlier studies of NC and thalamic GEs (Mak-McCully et al., 2017; Gonzalez et al., 2018) (i.e., TBs $\rightarrow$ DSs $\rightarrow$ SSs/USs, and HC-SSs tends to coincide with NC-SSs). Preferential cooccurrence, strong coherence, and phase-locking were noted between $\mathrm{pHC}$-SSs and NC-SSs in the posterior parietal and retrosplenial cortices.

\section{Characterization of human HC-SSs and SSRs in NREM}

We identified human HC-SWRs, SSRs, and SSs from intracranial recordings and aligned them with simultaneously recorded cortical events in NREM sleep, to determine whether their occurrences are related and have a consistent order, and whether their relations change between NREM Stages N2 and N3, or between $\mathrm{aHC}$ and $\mathrm{pHC}$ sites. Morphologically normal ripples were isolated from $>24 \mathrm{~h}$ continuous recordings in 20 SEEG patients with intractable focal epilepsy, with anterior (in 17 patients) and/or pHC contacts (in 11 patients), and related to sleep NC-
GEs in 12-30 bipolar recording channels per patient. Some ripples were found to co-occur with classical sharpwaves (Fig. 1A1; with across-patient mean LFP peak-to-peak amplitude of the sharp wave at $127.1 \mu \mathrm{V}$ ), and others did not (Fig. 1A2,A3; with peak amplitudes of 8.284 and $14.79 \mu \mathrm{V}$, respectively). The latter could sometimes be observed as HC-SSRs nestled within HC-SSs (Fig. $1 B, C$ ), as previously reported (Staresina et al., 2015). Timefrequency analysis triggering on HC-SWR ripple centers, SSR ripple centers (with all ripple centers found in the same HC-SSs included as separate trials), and HC-SS onsets (Fig. 1D) showed that, while HC-SWRs tended to be surrounded by a broad-band power decrease, HC-SSRs and HC-SSs did not. Similar to NC-SSs and HC-SWRs, HC-SSRs and HC-SSs in humans were mostly absent in waking (Fig. 1F; Extended Data Fig. 1-1E). Notably, HC-SSRs and HC-SSs did not appear to concentrate in NREM to the same extent as SWRs, in agreement with a recent report on human HC-SSs in REM (Lestra et al., 2018). However, whether these putative HC-SS hits in wake/REM are truly HC-SSs would be difficult to determine based on our NREM-based detection criteria, and further exploration of this phenomenon would be outside the scope of our current study.

We also found that, while HC sites produce both HC-SWRs and HC-SSs/HC-SSRs (Table 2), the occurrence rates (no./min) of HC-SSRs were remarkably lower than those of HC-SWRs in aHC $\left(p=1.602 \times 10^{-7}\right.$ in N2, $p=7.682 \times 10^{-8}$ in N3; twotailed paired $t$ tests); no similar difference was observed for $\mathrm{pHC}$, however $(p=0.4911$ in N2; $p=0.8038$ in N3; two-tailed paired $t$ tests) (Fig. 1E; Extended Data Fig. 1-1A-D. To verify that the relative imbalance of aHC SSRs and SWRs could not be explained by interpatient variance alone, we built LME models with HC-GE occurrence rates as response variable, and GE type (SWRs or SSRs), $\mathrm{aHC} / \mathrm{pHC}$, and NREM stage (N2/N3) as categorical predictor variables, with patient identity as random effect. We found that, indeed, there is a significant interaction effect of GE type $\times \mathrm{aHC} / \mathrm{pHC}$ on the occurrence rate of HC-GEs $\left(p=7.246 \times 10^{-7}\right)$.

HC-SSs/SSRs tended to occur more often in $\mathrm{pHC}$ than in aHC ( $p=0.0018 / 0.0002$ in N2, $p=0.0027 / 0.0004$ in N3, two-tailed two-sample $t$ tests) (Fig. 1E; Extended Data Fig. 1-1A-D). Transitional forms ("SXR") that were detected as both SWRs and SSRs constituted a minority of SSRs $(21.4 \%$ in N2 aHC, $26.9 \%$ in N3 aHC, $9.7 \%$ in $\mathrm{N} 2 \mathrm{pHC}, 13.0 \%$ in $\mathrm{N} 3 \mathrm{pHC}$ ), and the proportion of SXR among SSRs was greater in aHC than in $\mathrm{pHC}(p=0.0045$ for $\mathrm{N} 2, p=0.0060$ for N3, two-tailed two-sample $t$ tests), in line with the SWR rate reduction in pHC. Notably, while cortical spindles tend to occur more often in N2, we observed an increase of pHCSSs/SSRs in N3 $(p=0.0061$ and $p=0.0088)$, but not for aHCSSs/SSRs ( $p=0.0857$ and $p=0.0752$; two-tailed paired $t$ tests). To verify that differences in HC-SS/SSR occurrence rates across hippocampal origin and/or NREM stages could not be explained by interpatient variance alone, we built LME models with HC-GE occurrence rates as response variable, with $\mathrm{aHC} / \mathrm{pHC}$ and NREM stage as categorical predictor variables and patient identity as random effect. As expected, hippocampal longitudinal origin showed a significant effect on HC-GE rate $\left(p=0.0003\right.$ and $p=1.925 \times 10^{-5}$ for SSRs and HC-SSs), whereas the effect of NREM stage on HC-GE rate was nonsignificant $(p=0.4785$ and $p=0.2522$ for SSRs and $\mathrm{HC}-\mathrm{SSs}$ ). While there was no significant interaction effect between aHC/pHC and NREM stage for HC-SSRs $(p=0.1349)$, HC-SSs did show a significant interaction $(p=0.0324)$.

NREM Stage N3, associated with DSs and USs (which are in turn associated with SWRs), tends to be relatively more concentrated in earlier sleep cycles, whereas Stage N2, associated with SSs and thus SSRs, tends to occur in later cycles. Consequently, 


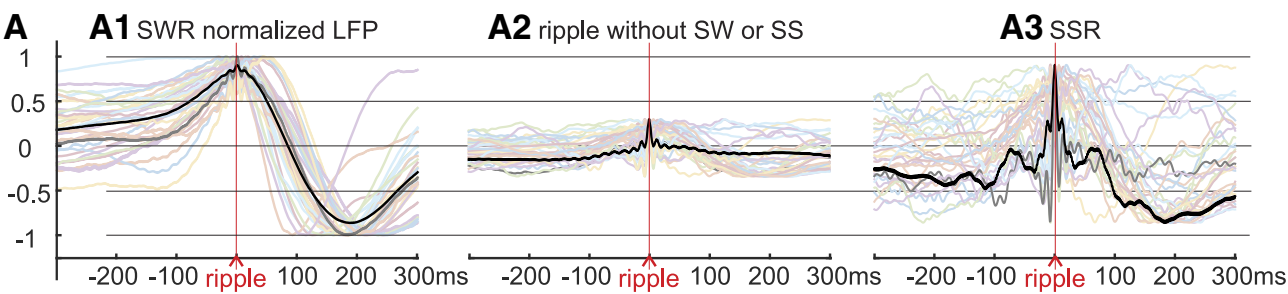

B B1 Example posterior HC LFP

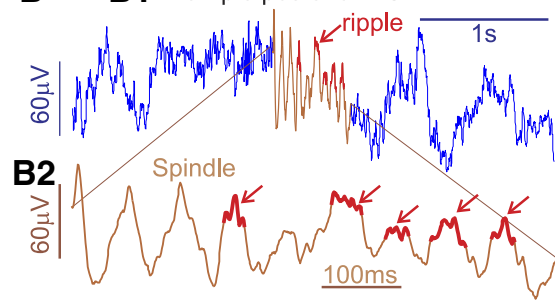

C C1 Spectral power triggered on spindle peak

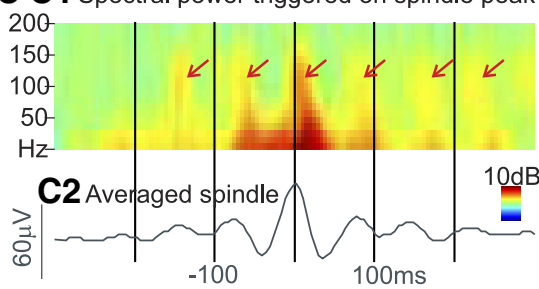

D

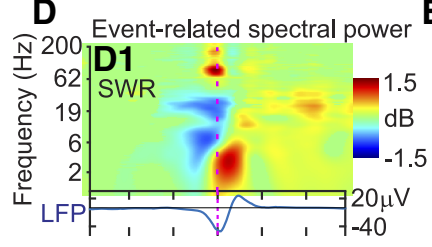

200
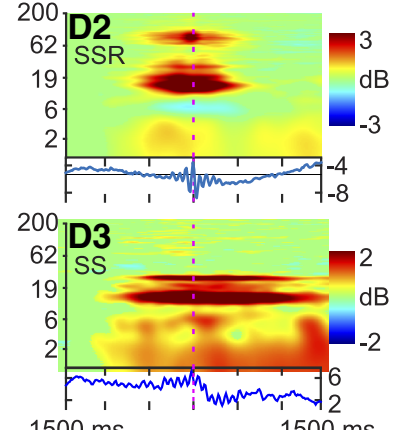

E

E1 Mean Rate of SWR, SSR and SS during N2

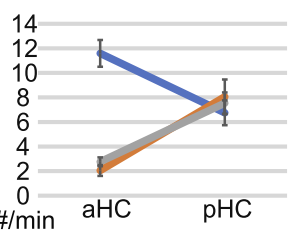

E2 Mean Rate of SWR, SSR and SS during N3

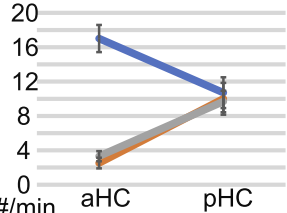
$\stackrel{0}{\# / m i n} \mathrm{aHC} \quad \mathrm{pHC}$ $-1500 \mathrm{~ms}$

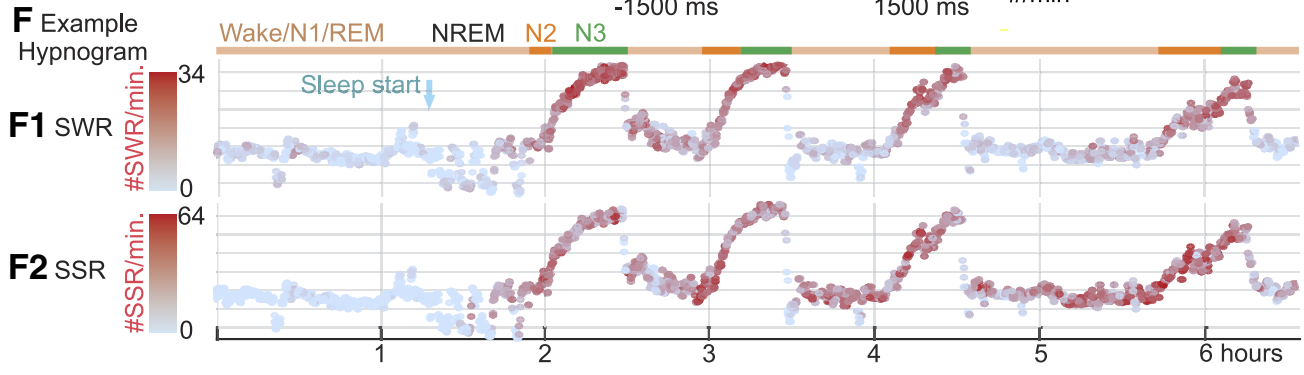

Figure 1. HC-SSs coordinate ripples and are found predominantly in PHC. A, Overlaid average waveforms of HC-SWR (A1), of ripples not coupled to sharpwaves (A2), and of HC-SSR (A3) across hippocampal contacts from 32 different patients, with one representative patient bolded in black for clarity. The non-normalized, across-patient mean LFP peak-to-peak amplitude of the sharp wave ( $\boldsymbol{A}$, black trace) is $127.1 \mu \mathrm{V}$, much greater than the corresponding peak amplitudes of 8.284 and $14.79 \mu \mathrm{V}$ in $\boldsymbol{B}$ and $\boldsymbol{C}$, respectively. $\boldsymbol{B}$, Example LFP trace of a $\sim 14 \mathrm{~Hz} \mathrm{HC}-\mathrm{SS}$ (red section) from bipolar recording in the $\mathrm{PHC}(\boldsymbol{B} 1)$, with expanded time-base plot of the spindle shown in $B 2 ; 80-90 \mathrm{~Hz}$ ripples occur preferentially at some peaks of the spindle waves. $C$, Example averaged time-frequency plot triggered on the center peak of HC-SSs from the same channel in $\boldsymbol{B}(\boldsymbol{C} 1, n=1086)$ and the corresponding average LFP (C2). D, Event-related spectral power is contrasted for SWRs (D1), SSRs (D2), and SSs (D3) from the same HC site, with corresponding average LFP traces below the time-frequency plots. The high-frequency ripple burst is seen for SWRs and SSRs but not prominently so in SSS. Prolonged spindle activity is seen for SSRs and SSs but not SWRs. E, HC-SWRs preferentially occur in aHC, whereas HC-SSS/SSRs prefer pHC. Each panel marks mean rates of HC-SWR/SSR/SS occurrence across patients in N2 (E1) or N3 (E2), for aHC and pHC. F, Example state plots showing the separation of NREM sleep Stages N2 and N3 from waking/N1/REM in $\sim 6.5 \mathrm{hLFP}$ recording, using the first principal component derived from vectors (one per cortical bipolar SEEG channel) of frequency power ratios (0.5-3 Hz over 0.5-16 Hz) (Gervasoni et al., 2004; Jiang et al., 2017). SWR rate (F1) and SSR rate (F2) are color coded with red intensity, and N2/N3 periods are marked with orange/green horizontal lines, respectively. Light blue arrow indicates the beginning of NREM sleep. Similar illustration for HC-SSs rate over time can be found in Extended Data Figure 1-1. E, Error bars indicate SEM.

we hypothesized that the HC-SSR to HC-SWR ratio would increase over the course of the night. We calculated in each NREM sleep segment the ratio of SSR counts to SWR counts, and compared these ratios in the first and second half of sleep. We found that, while both SWR and SSR occurrence rates decreased in the second half, the extent of the decrease was much more pronounced for HC-SWRs (Extended Data Fig. 1-1F). Indeed, the SSR/SWR ratio increased significantly from the first half of NREM sleep to the second half ( 0.67 vs $0.88, p=0.0363$, one-tailed paired $t$ test). However, when we accounted for patient-wise variability by introducing an LME model, we found the effect of early/late NREM on SSR/SWR ratio to be nonsignificant ( $p=0.5667)$.

Given the presence of both HC-SWRs and HC-SSRs in the same hippocampus (Table 2), and the preference of HC-SSRs to occur in pHC compared with aHC (Fig. 1E), we investigated whether a consistent relationship exists between SWRs and SSRs in aHC and pHC. In 2 of 8 subjects with both aHC and pHC contacts in the same $\mathrm{HC}$, we found significant elevations in $\mathrm{pHC}$ SSR counts at 100-500 ms following aHC-SWR triggers $(p<$ $7.811 \times 10^{-12}$ for all panels in Fig. $2 A 1$ [N2]; Extended Data Fig. $2-1 F$ [N3]; one-sample $t$ test, with SXR excluded), in contrast to the usual $\pm 200 \mathrm{~ms}$ co-occurrence of pHC-SWRs with aHCSWRs (Fig. 2A2; Extended Data Fig. 2-1G).

Since HC-SSs could co-occur with multiple ripples in different spindle cycles (Fig. 1B), we tallied the numbers of $\mathrm{HC}$ ripples within the duration of HC-SSs. Up to 9 ripples in N2 (or 10 in N3) could occur within one HC-SS (Fig. 2B; Extended Data Fig. 2-1H-J. Given the potential locality of HC ripples (Patel et al., 2013) and the SS phase modulation of high $\gamma$ amplitude (Fig. 2C), these ripple counts might be underestimates if each spindle cycle could contain a ripple. More than half of the HC-SSs overlapped with at least one ripple from the same HC site. Among the pop- 
Table 2. Hippocampal SEEG electrode coverage and occurrence rates of hippocampal GEs

\begin{tabular}{|c|c|c|c|c|c|c|c|c|c|c|c|c|c|c|c|c|}
\hline Patient no. & $\mathrm{LaHC}$ & $\mathrm{LpHC}$ & RaHC & RpHC & $\begin{array}{l}\text { SWR rate, } \\
\text { N2 aHC } \\
\text { (/min) }\end{array}$ & $\begin{array}{l}\text { SWR rate, } \\
\text { N2 pHC } \\
\text { (/min) }\end{array}$ & $\begin{array}{l}\text { SWR rate, } \\
\mathrm{N} 3 \mathrm{aHC} \\
\text { (/min) }\end{array}$ & $\begin{array}{l}\text { SWR rate, } \\
\text { N3 pHC } \\
\text { (/min) }\end{array}$ & $\begin{array}{l}\text { SSR rate, } \\
\text { N2 aHC } \\
\text { (/min) }\end{array}$ & $\begin{array}{l}\text { SSR rate, } \\
\mathrm{N} 2, \mathrm{pHC} \\
(/ \mathrm{min})\end{array}$ & $\begin{array}{l}\text { SSR rate, } \\
\text { N3, aHC } \\
\text { (/min) }\end{array}$ & $\begin{array}{l}\text { SSR rate, } \\
\mathrm{N} 3, \mathrm{pHC} \\
\text { (/min) }\end{array}$ & $\begin{array}{l}\text { HC-SS rate, } \\
\mathrm{N} 2, \text { aHC } \\
(/ \mathrm{min})\end{array}$ & $\begin{array}{l}\text { HC-SS rate, } \\
\text { N2, pHC } \\
(/ \mathrm{min})\end{array}$ & $\begin{array}{l}\text { HC-SS rate, } \\
\text { N3, aHC } \\
(/ \min )\end{array}$ & $\begin{array}{l}\text { HC-SS rate, } \\
\mathrm{N} 3, \mathrm{pHC} \\
(/ \mathrm{min})\end{array}$ \\
\hline 1 & $x$ & & & & 11 & & 19 & & 6.3 & & 9.4 & & 5.8 & & 9 & \\
\hline 3 & $x$ & $x$ & & & 11 & 12 & 24 & 25 & 1.4 & 3.4 & 1.4 & 5.6 & 2.8 & 5.4 & 2.6 & 9.3 \\
\hline 4 & $x$ & $x$ & $x$ & & 10 & 4.3 & 11 & 4.3 & 1.4 & 3.8 & 1.1 & 1.6 & 2.6 & 5.5 & 2.4 & 3.2 \\
\hline 5 & & & $x$ & $x$ & 21 & 3.7 & 38 & 14 & 1.3 & 7 & 1.9 & 9.9 & 1.4 & 4 & 1.8 & 7.2 \\
\hline 8 & & & $x$ & & 19 & & 20 & & 1.2 & & 1.3 & & 1.4 & & 1.9 & \\
\hline 9 & $x$ & $x$ & & & 13 & 7.9 & 16 & 7.2 & 1.1 & 7.2 & 1.5 & 11 & 2.2 & 5.9 & 2.8 & 9.3 \\
\hline 10 & & & $x$ & & 13 & & 18 & & 2.7 & & 2.2 & & 2.7 & & 2.7 & \\
\hline 11 & $x$ & & $x$ & $x$ & 7.3 & 7.7 & 12 & 12 & 0.44 & 1.1 & 0.49 & 1.5 & 1.5 & 2.4 & 1.1 & 2.8 \\
\hline 12 & & & & $x$ & & 5.5 & & 7 & & 13.4 & & 19 & & 12 & & 17 \\
\hline 13 & $x$ & $x$ & $x$ & & 13 & 6.2 & 19 & 4.9 & 2.6 & 8 & 2.4 & 8.2 & 3.6 & 9.8 & 3 & 13 \\
\hline 18 & $x$ & & & & 7.8 & & 10 & & 1.2 & & 1.1 & & 1.9 & & 1.9 & \\
\hline 19 & $x$ & $x$ & & & 12 & 2.6 & 19 & 6.1 & 7.7 & 14 & 9.7 & 16 & 6.6 & 9.7 & 9.3 & 12 \\
\hline 20 & & & $x$ & & 11 & & 12 & & 1.3 & & 3 & & 2.9 & & 5.6 & \\
\hline Mean & & & & & 12 & 7 & 18 & 11 & 2.1 & 8.2 & 2.8 & 10 & 2.8 & 7.5 & 3.5 & 9.8 \\
\hline SD & & & & & 1.1 & 1.0 & 1.6 & 1.8 & 0.44 & 1.4 & 0.62 & 1.9 & 0.36 & 0.87 & 0.59 & 1.3 \\
\hline
\end{tabular}
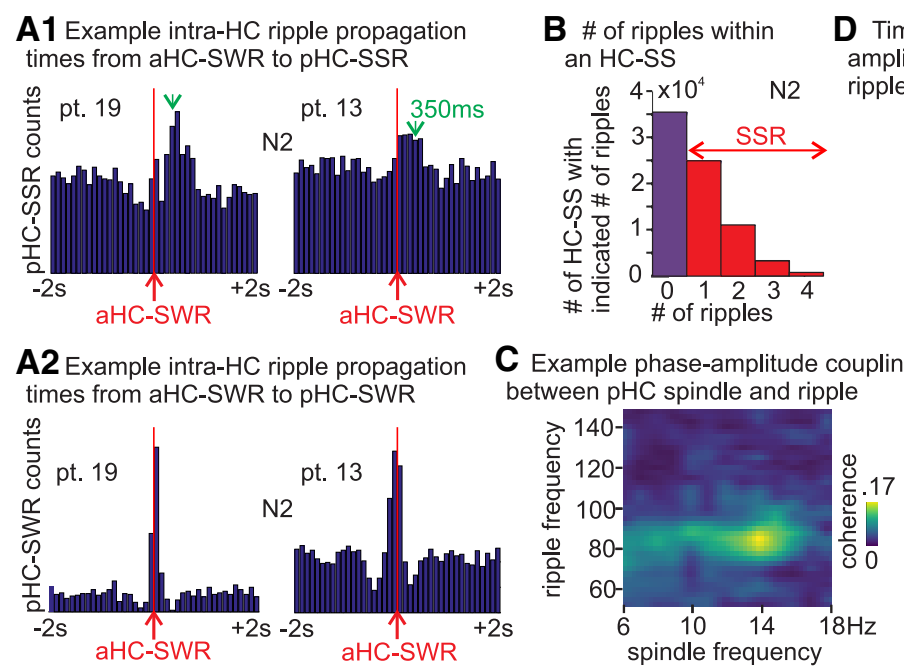

D Time-course of event-related phaseamplitude coupling between spindles and ripples recorded by the same electrode

Figure 2. Temporal relations of ripples and spindles, within and between hippocampal locations. A, Peristimulus time histograms with aHC-SWRs as triggers and either pHC-SSRs (A1) or pHC-SWRs (A2) as tallied events, with HC-SXR excluded from A1. In N2, pHC-SSRs tend to follow aHC-SWRs by $\sim 350 \mathrm{~ms}$ ( $\boldsymbol{A}$, green arrows), and aHC/pHC-SWRs tend to co-0ccur within $\pm 200 \mathrm{~ms}$. Similar results for N3 can be found in Extended Data Figure 2-1F, G. B, Histograms of ripple counts across all HC-SS in N2 (N3 results are in Extended Data Figure 2-1H. Not shown are the HC-SSs with $\geq 5$ ripples ( $n=256$ in N2, max 9). C, PAC within HC between HC-SS phase and ripple amplitude (bandpassed between 50 and $150 \mathrm{~Hz})$. D. The time course of intrahippocampal ERPAC between spindle $(10-16 \mathrm{~Hz})$ phase and ripple $(60-120 \mathrm{~Hz})$ amplitude, computed over all HC-SSs in NREM across all patients, separately for aHC (D1) and pHC (D2). Events are aligned at HC-SS starts (red vertical lines). Green line segments indicate the time segments with significant coupling $(p<0.001)$. The same pattern is observed if pHC events are randomly subselected to have the same number of events involved as aHC (Extended Data Figure 2-1E).

ulation of HC-SSs with at least one co-occurring ripple, $>60 \%$ had two or more ripples within the SS duration.

Time-frequency plots locked to HC spindle peaks yielded ripple power increases that appeared modulated by spindle frequency phase (Fig. 1D); this was further supported by intrahippocampal PAC (Fig. 2C). The time course of this coupling was examined by computing ERPAC between spindle $(10-16 \mathrm{~Hz})$ phase and ripple $(60-120 \mathrm{~Hz})$ amplitude, using previously published methods (Voytek et al., 2013). All HC-SSs in NREM across all patients were included in the computation, separated by longitudinal origin (aHC/pHC), with aligned events being HC-SS starts. As we expected from the PAC measure (Fig. 2C), we found significant event-related modulation of hippocampal ripple amplitude by HC-SS phase (Fig. $2 D$ ), with the effect being much more prominent in $\mathrm{pHC}$ (two-tailed paired $t$ test over mean ERPAC values from 0 to $1000 \mathrm{~ms}$ after trigger, $p<2.20 \times 10^{-16}$, Cohen's $\left.d=1.74\right)$.

\section{Co-occurrence of HC-SSs and HC-SSRs in anterior and posterior, or left and right $\mathrm{HC}$}

Although initial descriptions of SWRs in rodents emphasized their co-occurrence throughout the entire extent of both hippocampi (Buzsáki et al., 1992), more detailed studies showed that isolated SWRs are more common (Patel et al., 2013). The dominant and nondominant hippocampal formations of humans are thought to be 
specialized for verbal and visuospatial memory, respectively (Glosser et al., 1998), which might predict distinct memory traces and thus low bilateral co-occurrence of HC-SSs/SSRs. Analogously, the extent to which HC-SSRs and/or HC-SSs co-occur within the same hippocampus and/or bilaterally ought to be investigated.

We found that, for patients $(n=8)$ with both aHC and pHC contacts in the same HC, the overlap (i.e., co-occurrence within $50 \mathrm{~ms}$ ) between aHC-SSRs and pHC-SSRs (with N2 and N3 combined) was strikingly low, at $1.7 \pm 2.1 \%$, though still greater than the mean overlap percentage derived from chance $(p=0.0173$, one-tailed paired $t$ test). Similarly, while the overlap between aHC-SSs and pHC-SSs was significantly greater than chance $(p=$ 0.0010 , one-tailed paired $t$ test), the mean percentage remains low $(3.7 \pm 3.2 \%)$. Similarly, low overlap percentages were found when using data from N2 or N3 alone (Extended Data Fig. 2-1A).

We also evaluated HC-NC GE overlap in patients $(n=4)$ with bilateral (left and right) $\mathrm{HC}$ contacts, placed in either both anterior or both pHC. In these patients, co-occurrence of SSRs between HC in different hemispheres (with $\mathrm{N} 2$ and $\mathrm{N} 3$ combined) was minuscule $(0.41 \pm 0.47 \%)$. This was much less than that usually observed between two contacts within the same $\mathrm{HC}$, and not significantly greater than chance-derived overlap percentages ( $p=0.1506$, onetailed paired $t$ test). Co-occurrence of HC-SSs was also rare but more frequent than that of HC-SSRs $(1.0 \pm 0.66 \%)$. This small percentage nonetheless was significantly greater than chance ( $p=0.0062$, onetailed paired $t$ test). Similar overlap percentages were found when using data from N2 or N3 alone (Extended Data Fig. 2-1B).

Thus, in humans, a small but significant proportion of HC-SSRs and HC-SSs co-occur in both aHC and pHC, and only a minuscule (but still greater than chance for HC-SSs) proportion of HC-SSs and HC-SSRs co-occur in both left and right hippocampi. Furthermore, by varying the co-occurrence criterion range between 25 and 5000 $\mathrm{ms}$, we observed greater-than-chance co-occurrence rates for aHCand pHC-SSRs/SSs (as well as for left and right HC SSRs/SSs) initially (Extended Data Fig. 2-1A-D), but the actual co-occurrence rate tapered off exponentially (i.e., showed linear decay in log-log plots) toward chance level beyond $1000 \mathrm{~ms}$. Remarkably, the cooccurrence likelihood of aHC $+\mathrm{pHC}$ and bilateral HC-SSs/SSRs remains in an above-chance plateau until the time window in which co-occurrence is measured expands beyond $500-1000 \mathrm{~ms}$ for bilateral $\mathrm{HC}$ or for $\mathrm{aHC} / \mathrm{pHC}$. Since the typical significant latency (as estimated in Fig. 3) between NC-GEs and HC-SSs/SSRs is comparable, $\mathrm{HC}$-GEs from different $\mathrm{HC}$ regions could interact with disparate cortical regions at different times.

\section{Associations of cortical GEs with HC-SSRs and HC-SSs}

To examine the relationship between HC-SSs/SSRs and cortical activity, different cortical GEs (NC-GEs, including TBs, DSs, USs, and SSs) were identified in simultaneous recordings from all lobes. These recording channels were differential derivations between adjacent contacts spanning the cortical ribbon, and were thus assured of being locally generated. Detection algorithms have all been extensively validated in our previous studies (Mak-McCully et al., 2015, 2017; Gonzalez et al., 2018). To display the regularities in our data, we constructed histograms of the occurrence times of each NC-GE (TB onset, SS onset, DS peak, US peak) in relation to HC event triggers [HC-SWR ripple center, HC-SSR ripple center (multiple ripples within a single HC-SS counted as separate trials), and HC-SS onset] between each pair of HC $(n=32)$ and NC $(n=366)$ channels $(n=$ 20 patients). Separate analyses were performed for Stages N2 versus $\mathrm{N} 3$, for aHC versus $\mathrm{pHC}$, and for $\mathrm{HC}-\mathrm{NC}$ pairs in the same versus opposite hemispheres. Detailed breakdown of histogram contents by GE types and HC-SS/SSR sources (N2 vs N3, aHC vs pHC, ipsi- lateral vs contralateral) can be found in Extended Data Fig. 3-1. For both HC-SSs and HC-SSR, across all NC-GE types, in both N2 and $\mathrm{N} 3$, the HC-NC associations appeared to be more frequently involving $\mathrm{pHC}$ than $\mathrm{aHC}$, as reflected in the elevation of NC-GE counts (Extended Data Fig. 3-1; this agrees with our earlier observation that HC-SSs and HC-SSRs predominantly occurred in $\mathrm{pHC}$, and is in contrast with the distribution observed for SWRs Fig. 1E).

Of the 598 total HC-NC channel pairs, 555 (93\%) of all pairs $(429,94 \%$ of the ipsilateral pairs and $126,90 \%$ of the contralateral pairs) had at least one histogram showing a significant relationship between the HC-SSs/SSRs and a NC-GE at $p<0.0 .05$ after FDR correction (randomization test, see Experimental design and statistical analysis), suggesting widespread NC-GE involvement with HC-SSs/SSRs. The percentages of significant ipsilateral and contralateral pairs were not significantly different (Fisher's exact test, $p=0.1888$ ). Of the entire set of 4784 histograms involving HC-SSRs and NC-GEs, 1247 (26\%) showed a significant association; for those involving HC-SSs and NC-GEs, $2033(43 \%)$ showed a significant association, which is significantly greater than the proportion of histograms coupled to HCSSRs $\left(p=1.385 \times 10^{-64}\right.$, Fisher's exact test $)$. The significant histograms (for examples, see Fig. 3) include all types of NC-GEs from channels across all cortical lobes. For HC-SSRs, the peak of the histogram represented a mean increase of $157 \%$, or median increase of $86.7 \%$ above baseline (measured as the mean height of nonsignificant bins), with SD of $250 \%$, and positive skew value of 6.40. For HC-SSs, the peak of the histogram represented a mean increase of $147 \%$, or median increase of $91.3 \%$ above baseline, with SD of $220 \%$, and positive skew value of 9.19 .

We summarized these data further by constructing histograms of peak latencies from the histograms described above. Specifically, we determined the latency of peak occurrence rate relative to HC-SSR or HC-SS time for each significant HCNC-GE histogram, and plotted these as a histogram for each NC-GE type, separately for $\mathrm{N} 2$ versus $\mathrm{N} 3$ and aHC versus pHC (Fig. 4; Extended Data Fig. 4-1; Extended Data Fig. 4-2. As noted above, $\mathrm{N} 2$ and $\mathrm{pHC}$ sites had more HC-SSRs and HC-SSs. Results were also summarized by plotting each cortical channel after morphing to a standardized brain (Fig. 4; Extended Data Fig. 4-1; Extended Data Fig. 4-2. In the summary histograms, significant temporal relationships were found between HC-SSRs and each of the NC-GE types. As shown in Table 3, we tested for the presence of a significantly nonrandom distribution of NC-GE peak times with respect to HC-SSRs (column 4), and found significant results for both $\mathrm{N} 2$ and N3, and both aHC and pHC, for NC-SSs and NC-USs. NC-TBs and HCSSRs were significantly related in $\mathrm{N} 2$, but not in $\mathrm{N} 3$, possibly due to NC-TBs being more abundant and more related to NC-DSs in N2 (Gonzalez et al., 2018). NC-DSs and HC-SSRs were significantly related in $\mathrm{N} 2$ for both aHC and $\mathrm{pHC}$, but only for $\mathrm{pHC}$ in $\mathrm{N} 3$, which may reflect the preference of HC-SSRs to occur in pHC. Similarly, in the summary histograms, significant temporal relationships were found between HC-SS and each of the NC-GE types. We also tested for the presence of a significantly nonrandom distribution of NC-GE peak times with respect to HC-SSs (Table 3, column 8); we found significant results for both $\mathrm{N} 2$ and $\mathrm{N} 3$, and both $\mathrm{aHC}$ and $\mathrm{pHC}$, for NC-TBs, DSs, SSs, and USs.

We also examined whether the peak latency in the summary histograms between HC-SSs/SSRs and NC-GEs differed significantly from zero, and computed both the mean of the included HC-NC channel pairs (Table 3, columns 5 and 9) and the center of a distribution fitted over the peak latencies (Table 3, columns 6 and 10). Based on the fitted distribution centers in N2, NC-TB onsets significantly preceded aHC-SSRs by $741 \mathrm{~ms}$, NC-DS peaks 
A

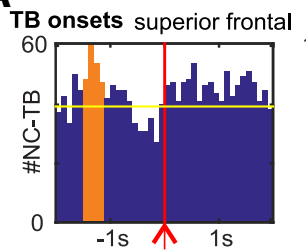

DS peaks
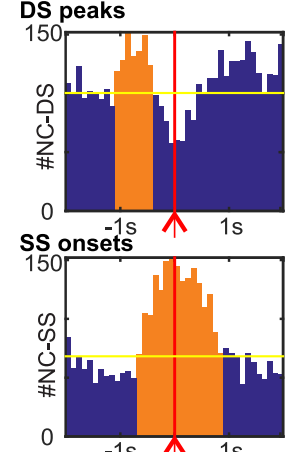

US peaks $-1 \mathrm{~s}$ 1s

US peaks

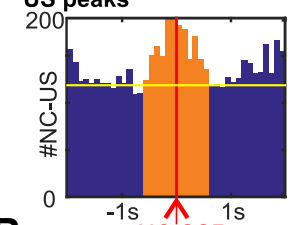

B

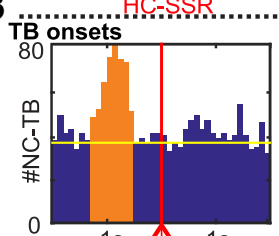

DS peaks

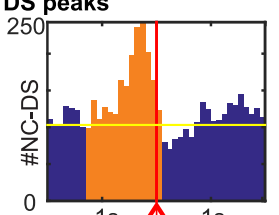

SS onsets

150

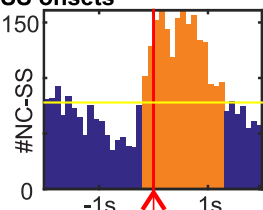

US peaks

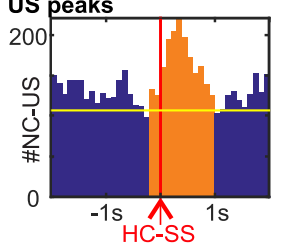

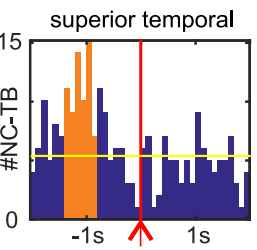
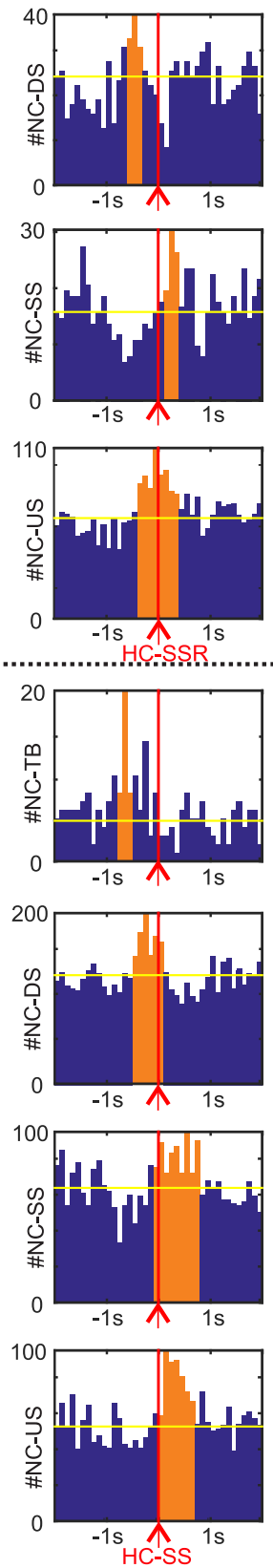
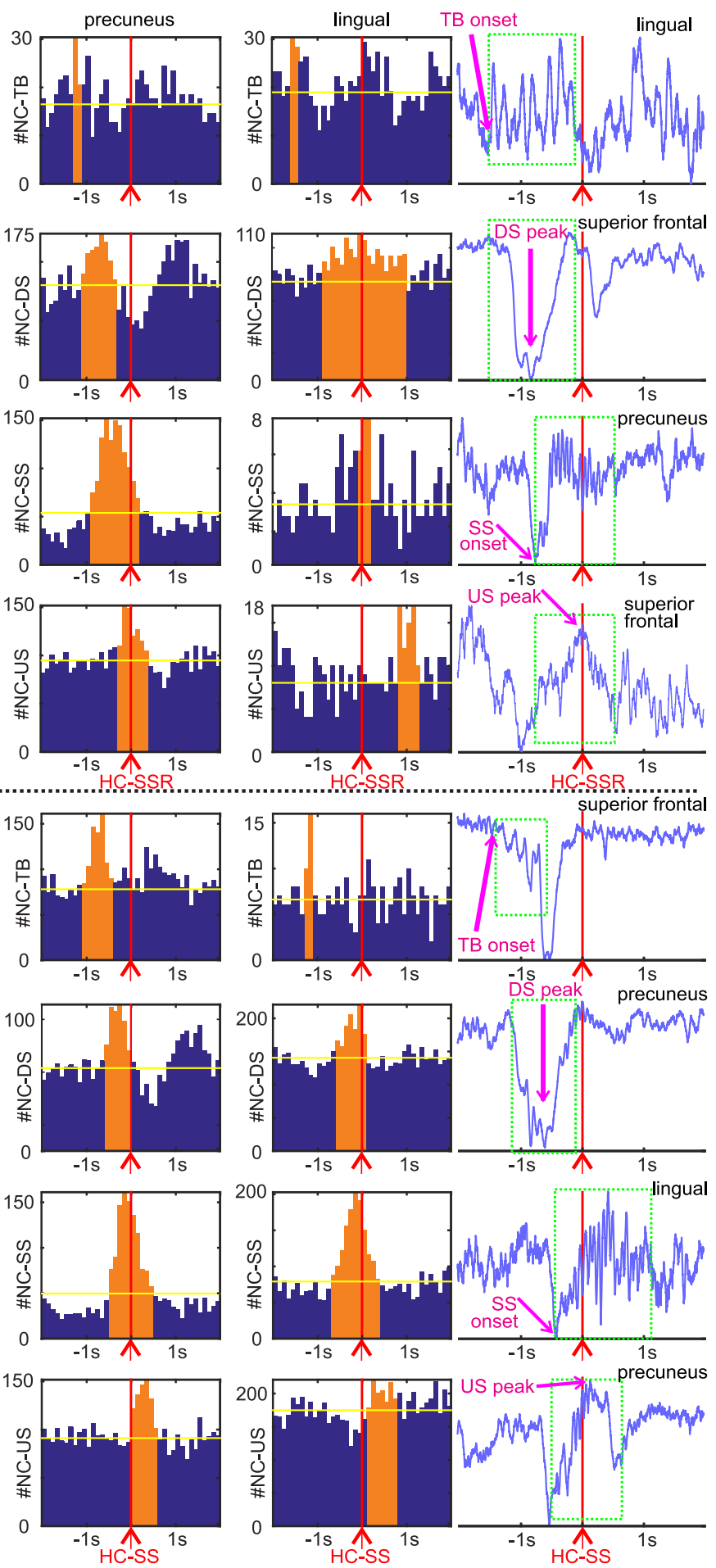

Figure 3. NC-GEs in relation to HC-SSRs $(\boldsymbol{A})$ and HC-SSs $(\boldsymbol{B})$, for example channel pairs. Each row of histograms is for a different type of NC-GE: TB onsets, DS peaks, SS onsets, and US peaks. Right, Example LFP trace containing the corresponding NC-GE (green dashed line boxes). Each column of plots shows example histograms with significant temporal correlations between HC-SSs/SSRs and NC-GEs from the same NC region in (from left to right) frontal, temporal, parietal, and occipital lobes. Magenta vertical lines indicate trigger (HC-SS/SSR) location for the peristimulus histograms. Orange bars represent the time ranges with both peak NC-GE occurrence rate and significant correlation. Yellow horizontal bars represent the average expected NC-GE counts from randomized control distributions. A summary of histogram contents can be found in Extended Data Figure 3-1.

significantly preceded by $575 \mathrm{~ms}$, NC-SS onsets preceded by 163 $\mathrm{ms}$, and NC-US peaks followed by $93 \mathrm{~ms}$. Generally, similar results could be found for pHC-SSRs in N2/N3, based on both mean peak latencies and fitted distribution peak centers (Table 3): at least among the NC-GEs that preceded HC-SSRs, there appeared to be a reliable sequence of $\mathrm{TB} s \rightarrow \mathrm{DSs} \rightarrow \mathrm{SSs}$ for both $\mathrm{aHC}$ and $\mathrm{pHC}$ in N2. Similarly, for $\mathrm{pHC}$ in N3, the $\mathrm{TB} \rightarrow \mathrm{DS} \rightarrow \mathrm{SS}$ sequence persisted. Finally, while NC-USs were significantly coupled to HC-SSRs across $\mathrm{N} 2$ and $\mathrm{N} 3$ for both aHC and $\mathrm{pHC}$, there were no significant biases to precede or follow HC-SSRs, indicat- 

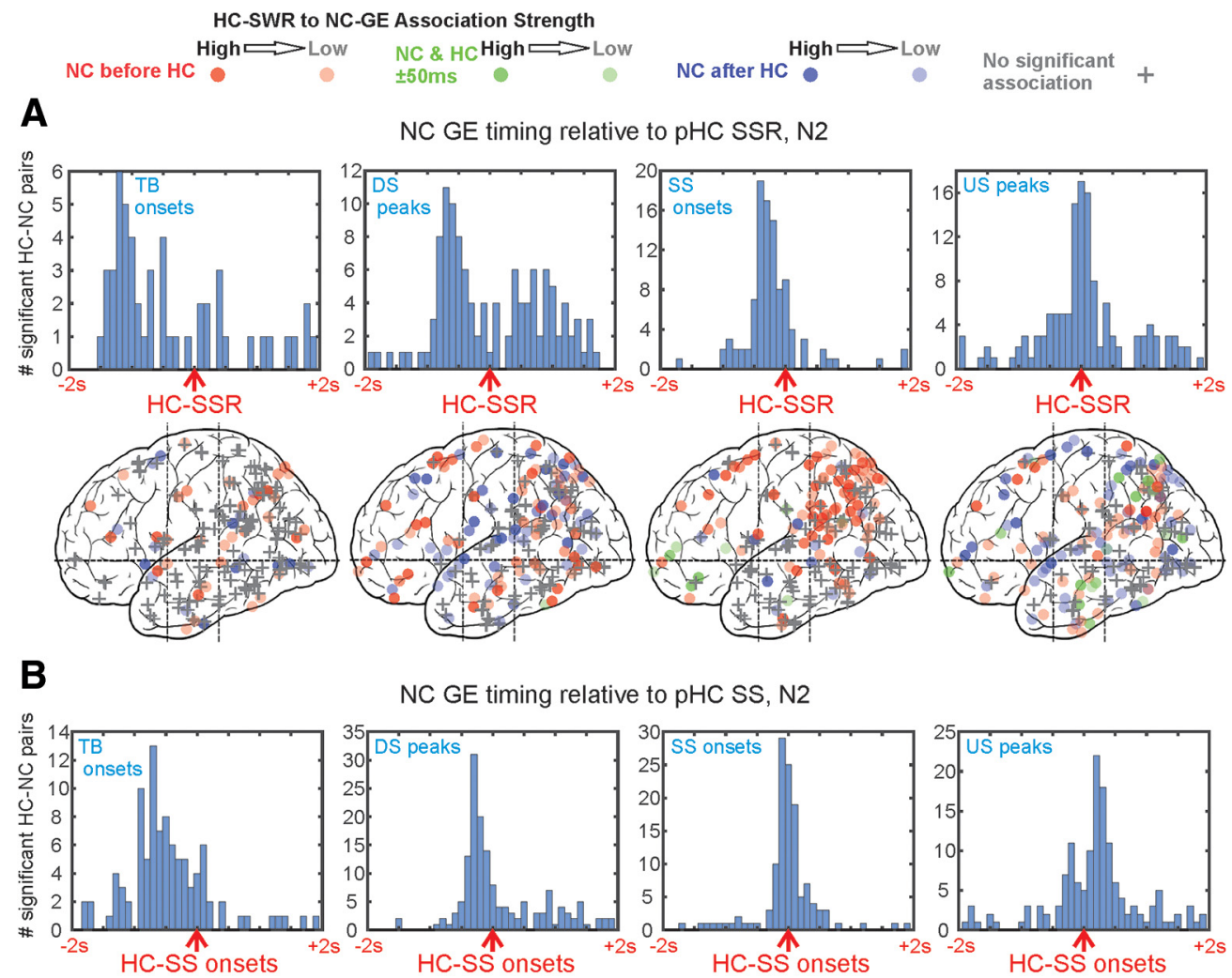

NC GE timing relative to $\mathrm{pHC}$ SS, N2
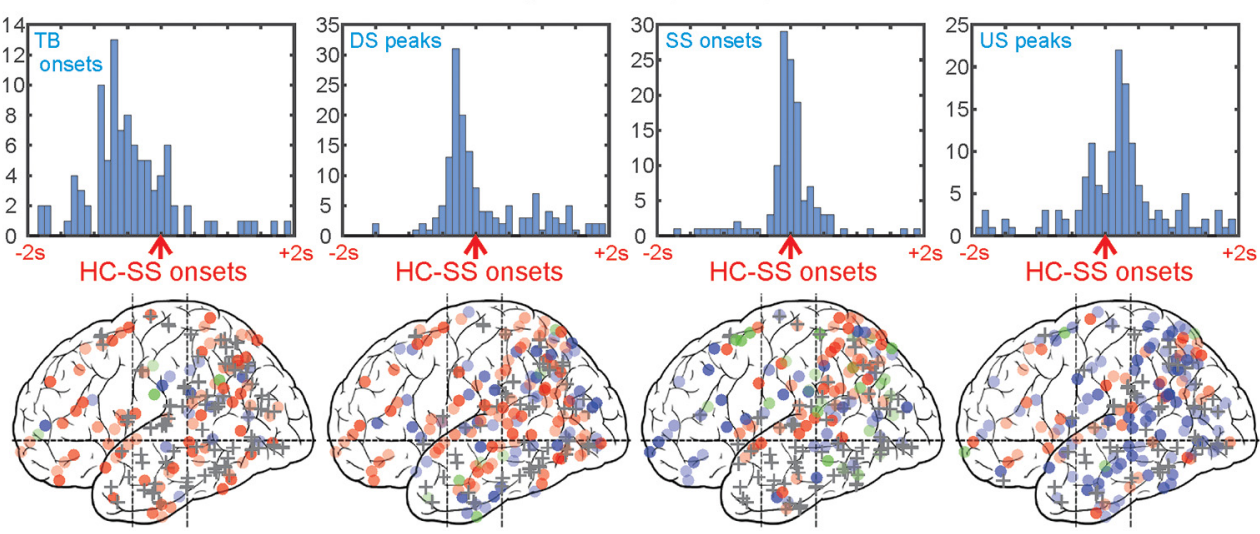

Figure 4. GEs across NC tend to occur with consistent latencies from HC-SSRs $(\boldsymbol{A})$ and HC-SSs $(\boldsymbol{B})$. $A$, In N2, with pHC-SSRs as reference, NC-GEs tend to occur in the following order: TB starts $\rightarrow$ DS peaks $\sim=S S$ starts $\rightarrow$ US peaks; NC-US peaks tend to co-occur with HC-SSRs. Similar results for pHC in N3 and for aHC in N2/N3 can be found in Extended Data Figure 4-1, and equivalent results for pHC in N2 with only the first SSR in each HC-SS as trigger can be found in Extended Data Figure 4-3. B, In N2, with pHC-SS as reference, NC-GEs tend to occur in the following order: TB starts $\rightarrow$ DS peaks $\rightarrow$ SS starts $\rightarrow$ US peaks. NC-SSs tend to C0-0ccur with HC-SSs, and widespread NC-USs tend to follow. Similar results for pHC in N3 and for aHC in N2/N3 can be found in Extended Data Figure 4-2. A, B , Top rows contain histograms of peak latencies for HC-NC channel pairs with significant temporal correlations between HC-GEs and NC-GES. Each count is the peak latency of a particular HC-NC channel pair. Bottom rows contain maps of peak latency between HC-GEs and NC-GEs for cortical channels. Circles represent where NC-GE relationships with HC-SSRs/SSs are significant. Plus signs indicate nonsignificant channels. The intensity of each circle corresponds to the strength of HC-SSR to NC-GE coupling estimated. The color of each circle representss peak latency: Red represents NC-GEs before HC-SSRs. Blue represents NC-GEs after HC-SSRs. Green represents NC-GEs C0-0ccurring with HC-SSRs (i.e., within 50 ms of each other). Both hemispheres and medial and lateral cortical sites are superimposed in each plot. Additional statistical results on the nonhomogeneity of HC-coupled NC site distributions can be found in Extended Data Figure 4-4, Extended Data Figure 4-5, and Extended Data Figure 4-6.

ing that NC-USs tended to co-occur with HC-SSRs and were likely to be the last in the sequence of NC-GE types we observed (i.e., TBs $\rightarrow$ DSs $\rightarrow$ SSs $\rightarrow$ USs). This sequence appears also in relation to HC-SSs (Table 3, columns 8-11). These observations are consistent with the sequence previously established in earlier studies of NC and thalamic GEs (Mak-McCully et al., 2017; Gonzalez et al., 2018).

HC-NC GE co-occurrence patterns are anatomically selective Having established that SSRs in particular HC sites are significantly associated with GE in NC sites, we asked whether cooccurring HC-SSRs/NC-GEs were randomly distributed across NC locations for a given HC site (Extended Data Fig. 4-4). Across different GEs, for $\sim 70 \%$ of the HC sites, their SSRs co-occurred with GEs in a nonrandom distribution of NC sites in N2 $\left(\chi^{2}\right.$ test of homogeneity). Similar results were obtained for HC-SSs in N2 but were less striking in N3 (Extended Data Fig. 4-4; Extended Data Fig. 4-5).
Given that $\mathrm{HC}-\mathrm{NC}$ co-occurrences are anatomically nonrandom (i.e., selective), we further determined whether they differed between simultaneously recorded $\mathrm{aHC}$ and $\mathrm{pHC}$ sites by performing Wilcoxon signed rank tests to evaluate whether for a given GE type, the proportions of GEs from significantly coupled NC sites that overlapped with aHC-SSRs/SSs differ from the proportions for pHCSSRs/SSs. Each test required that both members of a HC site pair have a significant $\chi^{2}$ test result from 1. In total, $8 \mathrm{aHC} / \mathrm{pHC}$ pairs were examined. We found that, for all the qualified $\mathrm{aHC} / \mathrm{pHC}$ pairs, the distributions of significantly associated NC channels for aHC and for pHC differed significantly (Extended Data Fig. 4-6). Thus, different anatomical distributions of $\mathrm{NC}$ are significantly coupled with simultaneously recorded aHC and pHC.

\section{Differences in the NC-GE to HC-SSR relationship across NC} regions, NREM stages, HC sites, and GEs

While the summaries in Figure 4 and Table 3 gave us an overview of NC-GE and HC-SS/SSR relations, we noted that there appeared to 
Table 3. Relation of NC-GE to HC-SSR and HC-SS occurrence times ${ }^{a}$

\begin{tabular}{|c|c|c|c|c|c|c|c|c|c|c|}
\hline $\begin{array}{l}\text { NC-GE } \\
\text { type }\end{array}$ & $\begin{array}{l}\text { Sleep } \\
\text { stage }\end{array}$ & $\begin{array}{l}\mathrm{HC} \\
\text { longitudinal } \\
\text { axis }\end{array}$ & $\begin{array}{l}\text { NC-GE } \\
\text { versus HC-SSR } \\
\text { related (KS test, } \\
p \text { value) }\end{array}$ & $\begin{array}{l}\text { Peak } \\
\text { latency } \\
\text { from HC-SSR } \\
\text { (ms), mean }\end{array}$ & $\begin{array}{l}\text { Peak } \\
\text { latency } \\
\text { from HC-SSR } \\
\text { (ms), DistPeak }\end{array}$ & $\begin{array}{l}\text { Non-zero } \\
\text { latency } \\
\text { from HC-SSR } \\
\text { (binomial } \\
p \text { value) }\end{array}$ & $\begin{array}{l}\mathrm{NC}-\mathrm{GE} \\
\text { versus } \\
\mathrm{HC}-\mathrm{SS} \text { related } \\
\text { (KS test, } \\
p \text { value) }\end{array}$ & $\begin{array}{l}\text { Peak } \\
\text { latency } \\
\text { from HC-SS } \\
\text { (ms), mean }\end{array}$ & $\begin{array}{l}\text { Peak } \\
\text { latency } \\
\text { from HC-SS } \\
\text { (ms), DistPeak }\end{array}$ & $\begin{array}{l}\text { Non-zero } \\
\text { latency } \\
\text { from HC-SS } \\
\text { (binomial } \\
p \text { value) }\end{array}$ \\
\hline \multirow[t]{4}{*}{ TB onsets } & \multirow[t]{2}{*}{ N2 } & Anterior & 0 & -398 & -741 & 0 & 0 & -164 & -392 & 0 \\
\hline & & Posterior & 0.002 & -386 & -1074 & 0.011 & 0 & -455 & -698 & 0 \\
\hline & \multirow[t]{2}{*}{ N3 } & Anterior & 0.129 & 123 & 206 & 1 & 0 & -96 & -338 & 0.006 \\
\hline & & Posterior & 0.654 & -131 & -346 & 0.388 & 0 & -512 & -646 & 0 \\
\hline \multirow[t]{4}{*}{ DS peaks } & \multirow[t]{2}{*}{ N2 } & Anterior & 0 & -185 & -575 & 0 & 0 & 55 & -65 & 0.232 \\
\hline & & Posterior & 0 & 38 & 31 & 0.519 & 0 & 105 & -167 & 0.001 \\
\hline & \multirow[t]{2}{*}{ N3 } & Anterior & 0.216 & -95 & -426 & 0.117 & 0 & 148 & -57 & 1 \\
\hline & & Posterior & 0 & -288 & -489 & 0.001 & 0 & -64 & -184 & 0 \\
\hline \multirow[t]{4}{*}{ SS onsets } & \multirow[t]{2}{*}{$\mathrm{N} 2$} & Anterior & 0 & -16 & -163 & 0.213 & 0 & 80 & 73 & 0.086 \\
\hline & & Posterior & 0 & -211 & -353 & 0 & 0 & -19 & -106 & 0.368 \\
\hline & \multirow[t]{2}{*}{ N3 } & Anterior & 0.017 & -32 & -142 & 0.728 & 0.003 & -10 & 12 & 0.377 \\
\hline & & Posterior & 0 & -241 & -425 & 0 & 0 & -45 & -32 & 0.013 \\
\hline \multirow[t]{4}{*}{ US peaks } & \multirow[t]{2}{*}{ N2 } & Anterior & 0 & 35 & 93 & 0.221 & 0 & 348 & 534 & 0 \\
\hline & & Posterior & 0 & 68 & 62 & 0.643 & 0 & 165 & 212 & 0 \\
\hline & \multirow[t]{2}{*}{ N3 } & Anterior & 0.013 & -131 & -180 & 0.609 & 0 & 338 & 568 & 0 \\
\hline & & Posterior & 0 & -49 & -71 & 0.699 & 0 & 199 & 111 & 0 \\
\hline
\end{tabular}

a Separate values and statistical significance tests are shown for NREM (sleep stages N2 vs N3), aHC versus pHC, and for different NC GEs (TBs, SSs, DS, USs). Tests indicate whether there was a significant association between the times of occurrence of the HC-SSRs/HC-SSs and GEs (fourth and eighth columns); if the NC-GEs occurred significantly before the HC-SSRs/HC-SSs (negative numbers) or before after, when measuring the peak latency as the mean value across cortico-HC pairs (fifth and ninth columns), or as the peak of an extreme value distribution fitted over the $\pm 2000 \mathrm{~ms}$ histogram-of-histograms in Figure $4 A$ and Extended Data Figure $4-1$ (sixth column) or in Figure $4 B$ and Extended Data Figure 4-2 (10th column). The seventh and 11th columns show whether there is a significant difference in the number of NC-GEs occurring 2000 ms before versus $2000 \mathrm{~ms}$ after the HC-SSRs/HC-SSs. Extremely small ( $<0.0001) p$ values are represented by 0 for clarity. While NC-DS was significantly earlier than pHC-SSR in N3, but not in N2, it should be noted that NC-DS distribution appeared bimodal for N2 pHC-SSR, which likely distorted the mean and distribution peak estimations: the larger pretrigger peak of the NC-DS latency distribution for N2 pHC-SSR, like the NC-DS distribution peak in N3, preceded that of the NC-SS latency distribution ( 700 ms pre-SSR for NC-DS vs 353 ms for NC-SS) (Fig. 4 A).

Table 4. Numbers of HC-NC channel pairs by NC ROIs, and proportions of HC-NC channel pairs with significant NC-GE/HC-SSR relationships across NC ROIs ${ }^{a}$

\begin{tabular}{|c|c|c|c|c|c|c|c|c|c|c|c|c|c|c|c|c|c|c|}
\hline \multirow{4}{*}{$\begin{array}{l}\text { NC-GE type } \\
\text { ROI }\end{array}$} & \multicolumn{18}{|c|}{ Proportion of channels showing significant NC-GE c0-occurrence with HC-SSR } \\
\hline & \multirow{2}{*}{\multicolumn{2}{|c|}{$\begin{array}{l}\text { Total no. of } \\
\text { channel pairs }\end{array}$}} & \multicolumn{4}{|l|}{$\mathrm{TB}$} & \multicolumn{4}{|l|}{ DS } & \multicolumn{4}{|l|}{ SS } & \multicolumn{4}{|l|}{ US } \\
\hline & & & \multicolumn{2}{|l|}{ N2 } & \multicolumn{2}{|l|}{ N3 } & \multicolumn{2}{|l|}{$\mathrm{N} 2$} & \multicolumn{2}{|l|}{ N3 } & \multicolumn{2}{|l|}{ N2 } & \multicolumn{2}{|l|}{ N3 } & \multicolumn{2}{|l|}{ N2 } & \multicolumn{2}{|l|}{ N3 } \\
\hline & $\mathrm{aHC}$ & $\mathrm{pHC}$ & $\mathrm{aHC}$ & $\mathrm{pHC}$ & $\mathrm{aHC}$ & $\mathrm{pHC}$ & $\mathrm{aHC}$ & $\mathrm{pHC}$ & $\mathrm{aHC}$ & $\mathrm{pHC}$ & $\mathrm{aHC}$ & $\mathrm{pHC}$ & $\mathrm{aHC}$ & $\mathrm{pHC}$ & $\mathrm{aHC}$ & $\mathrm{pHC}$ & $\mathrm{aHC}$ & $\mathrm{pHC}$ \\
\hline Lateral occipital & 15 & 15 & 0.000 & 0.000 & 0.000 & 0.067 & 0.267 & 0.333 & 0.067 & 0.200 & 0.133 & 0.133 & 0.133 & 0.067 & 0.200 & 0.267 & 0.133 & 0.067 \\
\hline Medial temporo-occipital & 31 & 26 & 0.129 & 0.231 & 0.032 & 0.115 & 0.452 & 0.385 & 0.129 & 0.385 & 0.129 & 0.231 & 0.097 & 0.154 & 0.355 & 0.538 & 0.129 & 0.385 \\
\hline Lateral parietal & 74 & 51 & 0.135 & 0.176 & 0.081 & 0.039 & 0.297 & 0.451 & 0.203 & 0.353 & 0.216 & 0.549 & 0.041 & 0.373 & 0.473 & 0.529 & 0.176 & 0.353 \\
\hline Medial occipito-parietal & 34 & 27 & 0.118 & 0.259 & 0.059 & 0.037 & 0.176 & 0.630 & 0.206 & 0.370 & 0.324 & 0.630 & 0.059 & 0.556 & 0.412 & 0.556 & 0.176 & 0.333 \\
\hline Lateral temporal & 86 & 54 & 0.128 & 0.241 & 0.035 & 0.019 & 0.314 & 0.444 & 0.081 & 0.222 & 0.186 & 0.333 & 0.070 & 0.148 & 0.430 & 0.574 & 0.151 & 0.167 \\
\hline Insula & 11 & 6 & 0.273 & 0.333 & 0.182 & 0.000 & 0.636 & 0.500 & 0.273 & 0.167 & 0.091 & 0.500 & 0.000 & 0.167 & 0.364 & 0.667 & 0.364 & 0.333 \\
\hline Paracentral & 29 & 16 & 0.207 & 0.313 & 0.069 & 0.063 & 0.345 & 0.625 & 0.172 & 0.313 & 0.276 & 0.313 & 0.034 & 0.375 & 0.448 & 0.625 & 0.138 & 0.313 \\
\hline Orbitofrontal & 23 & 6 & 0.174 & 0.000 & 0.174 & 0.000 & 0.348 & 1.000 & 0.087 & 0.000 & 0.261 & 0.667 & 0.130 & 0.333 & 0.478 & 0.833 & 0.261 & 0.167 \\
\hline Prefrontal & 49 & 14 & 0.204 & 0.214 & 0.102 & 0.143 & 0.510 & 0.929 & 0.224 & 0.500 & 0.265 & 0.714 & 0.245 & 0.571 & 0.469 & 0.929 & 0.245 & 0.429 \\
\hline Cingulate & 13 & 6 & 0.077 & 0.333 & 0.077 & 0.167 & 0.385 & 0.500 & 0.385 & 0.500 & 0.308 & 0.667 & 0.308 & 0.500 & 0.462 & 0.667 & 0.231 & 0.667 \\
\hline Total no. of channel pairs across NC & 365 & 221 & & & & & & & & & & & & & & & & \\
\hline Average proportions & & & 0.144 & 0.210 & 0.081 & 0.065 & 0.373 & 0.580 & 0.183 & 0.301 & 0.219 & 0.474 & 0.112 & 0.324 & 0.409 & 0.618 & 0.200 & 0.321 \\
\hline SDs & & & 0.072 & 0.116 & 0.056 & 0.056 & 0.125 & 0.212 & 0.094 & 0.148 & 0.077 & 0.196 & 0.092 & 0.174 & 0.081 & 0.171 & 0.071 & 0.158 \\
\hline
\end{tabular}

${ }^{a}$ Details on Freesurfer parcellations included in each ROI can be found in Extended Data Table 4-1.

be regional differences across $\mathrm{NC}$ in the strength of temporal correlations. We also considered the possibility that "hot spots" may exist in $\mathrm{NC}$ and contribute to the widespread positive skewness in the distributions of GE counts across individual HC-NC GE histograms (Extended Data Fig. 3-1). We therefore explored the spatial distribution of HC-NC relationship further by tallying the proportion of significant $\mathrm{HC}-\mathrm{NC}$ channel pairs across different $\mathrm{NC}$ regions, with respect to different NC-GE types and different HC-SSR/SS sources (Table 4, Table 5). We then performed two four-way ANOVAs (one for HC-SSRs, another for HC-SSs) to compare the main effects of NC-GE type (TB, SS, DS, US), NREM stage (N2 vs N3), aHC or pHC origin of HC-SS/SSR, and NC ROIs (Fig. 5A; coverage listed in Extended Data Table 4-1), as well as the two-way and three-way interaction effects. All post hoc analyses were performed with Tukey's range test. We checked the normality assumption by conducting the Lilliefors test on the residuals, and the null hypothesis of normality could not be rejected ( $p=0.2058$ for HC-SSRs, $p=0.0935$ for HC-SSs). Both the ANOVA and post hoc analyses' results ( $F$ statistics and $p$ values) are summarized in Table 6 (for HC-SSRs) and Table 7 (for HC-SSs).

For the ANOVA performed over proportions of NC channels showing significant NC-GE to HC-SSR temporal correlations, all four main effects were significant. The main effect for GE was due to the mean proportions of SSR-responsive HC-NC channel pairs differing between all GE type pairs, except NC-DSs and NC-USs. The mean proportions were 0.1252 for TBs, 0.2822 for SSs, 0.3591 for DSs, and 0.3874 for USs (Fig. 5D). The NREM stage main effect was due to more HC-NC channel pairs being 
Table 5. Numbers of HC-NC channel pairs by NC ROIs, and proportions of HC-NC channel pairs with significant relationships between NC-GEs and HC-SSs

\begin{tabular}{|c|c|c|c|c|c|c|c|c|c|c|c|c|c|c|c|c|c|c|}
\hline \multirow{4}{*}{$\begin{array}{l}\text { NC-GE type } \\
\text { ROI }\end{array}$} & \multicolumn{18}{|c|}{ Proportion of channels showing significant NC-GE co-occurrence with HC-SS } \\
\hline & \multirow{2}{*}{\multicolumn{2}{|c|}{$\begin{array}{l}\text { Total no. of } \\
\text { channel pairs }\end{array}$}} & \multicolumn{4}{|l|}{ TB } & \multicolumn{4}{|l|}{ DS } & \multicolumn{4}{|l|}{ SS } & \multicolumn{4}{|l|}{ US } \\
\hline & & & \multicolumn{2}{|l|}{ N2 } & \multicolumn{2}{|l|}{ N3 } & \multicolumn{2}{|l|}{ N2 } & \multicolumn{2}{|l|}{ N3 } & \multicolumn{2}{|l|}{ N2 } & \multicolumn{2}{|l|}{ N3 } & \multicolumn{2}{|l|}{ N2 } & \multicolumn{2}{|l|}{ N3 } \\
\hline & $\mathrm{aHC}$ & $\mathrm{pHC}$ & $\mathrm{aHC}$ & $\mathrm{pHC}$ & $\mathrm{aHC}$ & $\mathrm{pHC}$ & $\mathrm{aHC}$ & $\mathrm{pHC}$ & $\mathrm{aHC}$ & $\mathrm{pHC}$ & $\mathrm{aHC}$ & $\mathrm{pHC}$ & $\mathrm{aHC}$ & $\mathrm{pHC}$ & $\mathrm{aHC}$ & $\mathrm{pHC}$ & $\mathrm{aHC}$ & $\mathrm{pHC}$ \\
\hline Lateral occipital & 15 & 15 & 0.200 & 0.133 & 0.133 & 0.000 & 0.200 & 0.600 & 0.067 & 0.467 & 0.133 & 0.200 & 0.133 & 0.133 & 0.467 & 0.333 & 0.400 & 0.600 \\
\hline Medial temporo-occipital & 31 & 26 & 0.194 & 0.269 & 0.161 & 0.231 & 0.613 & 0.500 & 0.194 & 0.423 & 0.194 & 0.192 & 0.129 & 0.154 & 0.581 & 0.538 & 0.258 & 0.538 \\
\hline Lateral parietal & 74 & 51 & 0.432 & 0.412 & 0.176 & 0.176 & 0.757 & 0.706 & 0.311 & 0.608 & 0.324 & 0.706 & 0.095 & 0.353 & 0.797 & 0.627 & 0.365 & 0.804 \\
\hline Medial occipito-parietal & 34 & 27 & 0.324 & 0.370 & 0.147 & 0.185 & 0.529 & 0.630 & 0.265 & 0.556 & 0.324 & 0.667 & 0.147 & 0.556 & 0.765 & 0.630 & 0.353 & 0.667 \\
\hline Lateral temporal & 86 & 54 & 0.442 & 0.463 & 0.163 & 0.148 & 0.733 & 0.722 & 0.349 & 0.444 & 0.221 & 0.444 & 0.070 & 0.093 & 0.791 & 0.722 & 0.302 & 0.389 \\
\hline Insula & 11 & 6 & 0.364 & 0.333 & 0.182 & 0.000 & 0.727 & 0.833 & 0.273 & 0.500 & 0.364 & 0.333 & 0.000 & 0.000 & 0.545 & 0.500 & 0.182 & 0.167 \\
\hline Paracentral & 29 & 16 & 0.414 & 0.438 & 0.138 & 0.188 & 0.655 & 0.500 & 0.241 & 0.563 & 0.172 & 0.688 & 0.103 & 0.188 & 0.586 & 0.750 & 0.138 & 0.438 \\
\hline Orbitofrontal & 23 & 6 & 0.739 & 1.000 & 0.435 & 0.167 & 0.783 & 1.000 & 0.565 & 0.167 & 0.261 & 0.833 & 0.087 & 0.333 & 0.870 & 0.833 & 0.478 & 0.500 \\
\hline Prefrontal & 49 & 14 & 0.531 & 0.714 & 0.367 & 0.286 & 0.694 & 0.857 & 0.449 & 0.571 & 0.367 & 0.714 & 0.163 & 0.571 & 0.694 & 0.857 & 0.490 & 0.714 \\
\hline Cingulate & 13 & 6 & 0.385 & 0.500 & 0.385 & 0.333 & 0.692 & 0.667 & 0.692 & 0.833 & 0.385 & 1.000 & 0.308 & 0.500 & 0.923 & 0.667 & 0.538 & 0.833 \\
\hline Total no. of channel pairs across NC & 365 & 221 & & & & & & & & & & & & & & & & \\
\hline Average proportions & & & 0.402 & 0.463 & 0.229 & 0.171 & 0.638 & 0.701 & 0.341 & 0.513 & 0.274 & 0.578 & 0.124 & 0.288 & 0.702 & 0.646 & 0.350 & 0.565 \\
\hline SDs & & & 0.150 & 0.230 & 0.111 & 0.101 & 0.162 & 0.151 & 0.174 & 0.159 & 0.086 & 0.258 & 0.075 & 0.194 & 0.144 & 0.151 & 0.126 & 0.193 \\
\hline
\end{tabular}

A cingulate
prefrontal
orbitofrontal
paracentral
insula

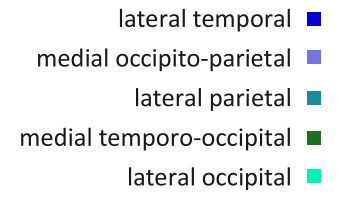

lateral occipital
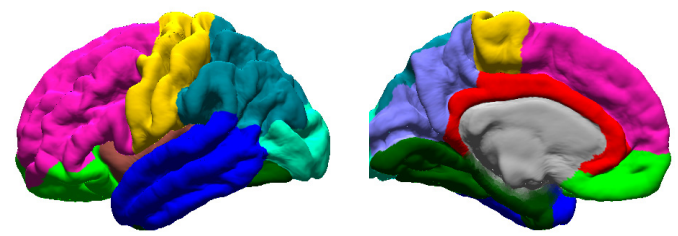

B ROI v. Sleep Stage v. HC axis, for HC-SSR $\mathrm{N} 2$ ant N2 post N3 ant N3 post

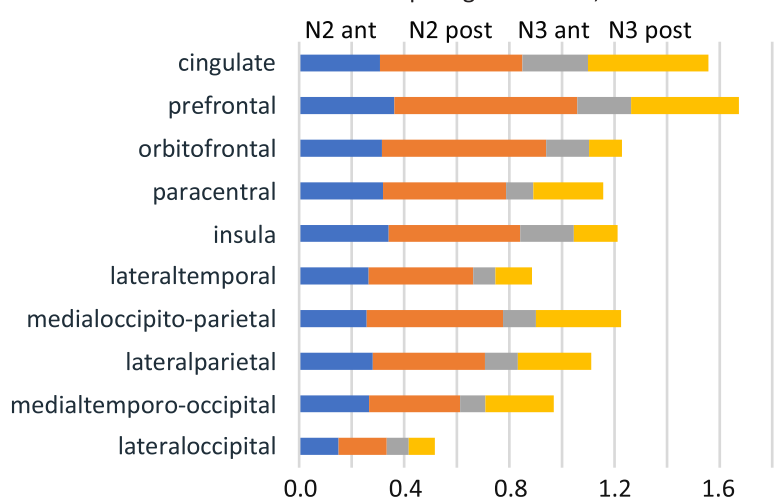

C ROI v. Sleep Stage v. HC axis, for HC-SS N2 ant N2 post N3 ant N3 post

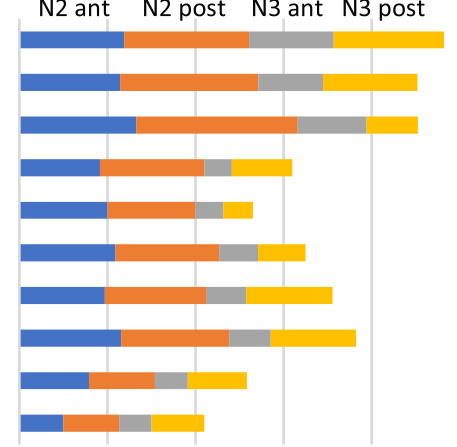

0.0

Proportion of coupled NC-HC channel pairs

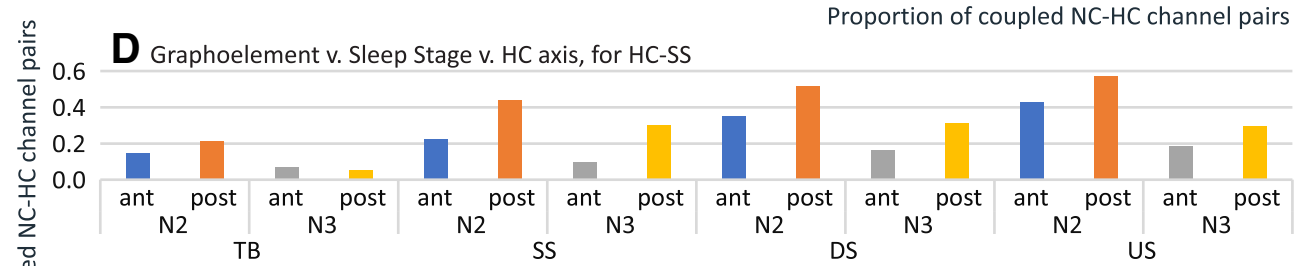

E Graphoelement v. Sleep Stage v. HC axis, for HC-SSR

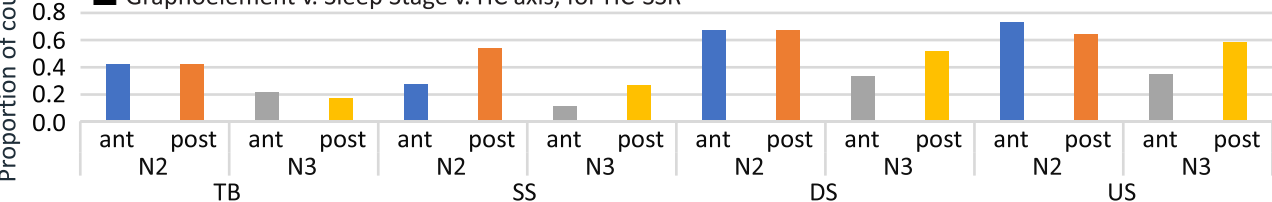

Figure 5. Strength of HC-NC association shows both correlativity and variability across GE types, NREM stages, HC-SS/SSR sources, and NC regions. $\boldsymbol{A}$, Map of NC ROIs used for analysis (Extended Data Table 4-1). B, C, Stacked bar graph of mean significant (evaluated as in Fig. 3) HC-NC channel pair proportions with regard to all channels found in each ROI. $\boldsymbol{B}$, Data were obtained by averaging across GE types for each SSR origin (different NREM stages and HC contact positions). C, Similarly, data were obtained by averaging across NC-GE types for each HC-SS origin. $\boldsymbol{D}, \boldsymbol{E}$, Global (i.e., across all NC ROIs) proportions of significant HC-NC channel pairs for HC-SSRs (D) and for HC-SSs $(\boldsymbol{E})$. $\boldsymbol{B}-\boldsymbol{E}$ share the same color legend. ant, aHC; post, pHC.

significantly related to HC-SSR in N2 (mean proportion of $0.3784)$ than in $\mathrm{N} 3(0.1985)$. The aHC/pHC main effect was due to more of NC being related to pHC-SSRs (0.3617) than to aHCSSRs (0.2152), consistent with our previous observation of higher
HC-SSR density in pHC than in aHC. The ROI main effect was due to a stronger HC-NC association by prefrontal locations, followed by cingulate, orbitofrontal, and medial occipitoparietal cortices; in contrast, lateral occipital and lateral temporal 
Table 6. Summary of four-way ANOVA comparing associations between HC-SSRs and NC-GEs ${ }^{a}$

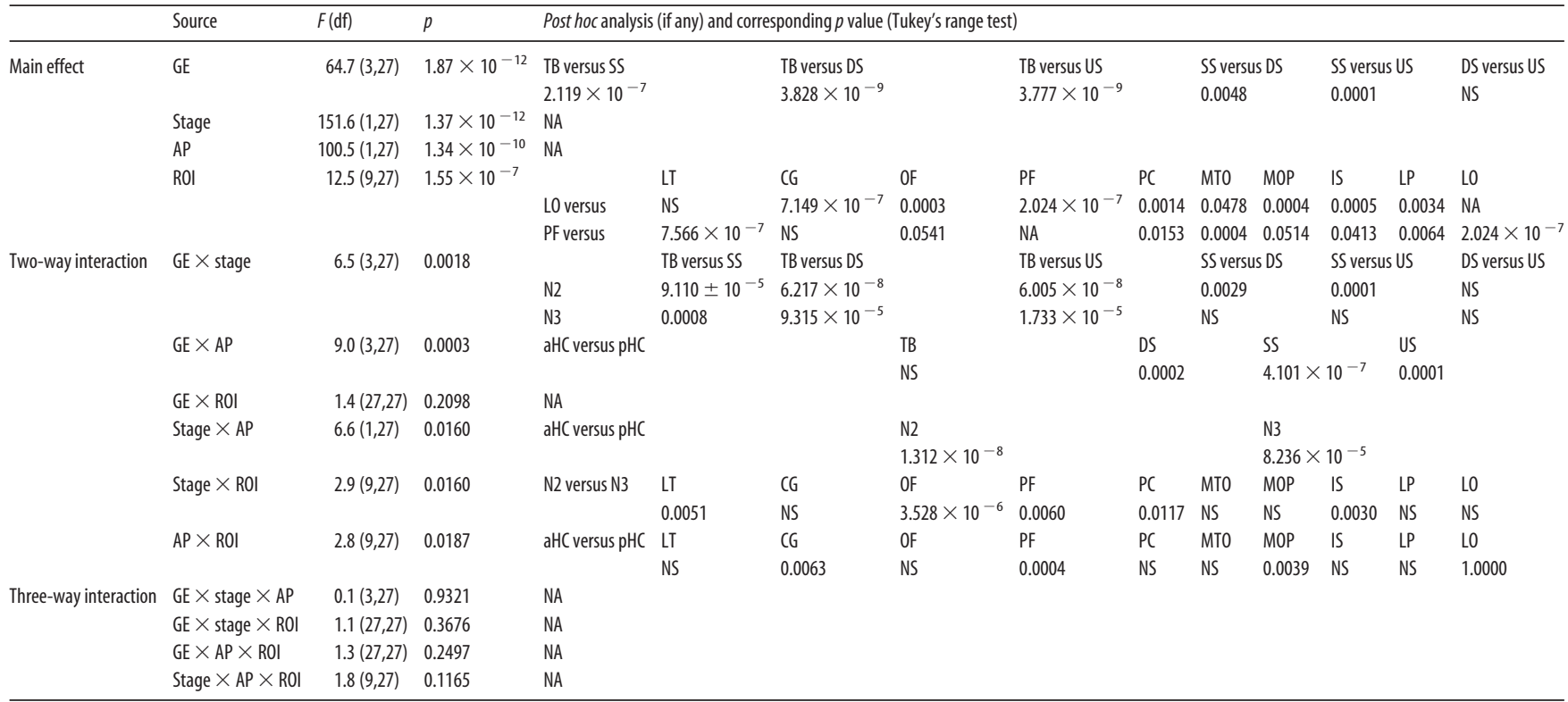

${ }^{a}$ For each HC-NC electrode pair and sleep stage (N2 vs N3), the significance of the NC-GE occurrence times relative to the HC-SS onset was determined (see Fig. 3A; Materials and Methods). We then tallied the proportion of significant channe pairs according to their HC location (aHC, pHC), NC location (see Extended Data Table 4-1; Fig. 5A), and GE type (TB, DS, SS, US). These proportions were then entered into ANOVA. L0, Lateral occipital; LT, lateral temporal; (G, cingulate; OF, orbitofrontal; PF, prefrontal; PC, paracentral; MT0, medial temporo-occipital; MOP, medial occipito-parietal; IS, insula; LP, lateral parietal; NS, not significant (for post hoc Tukey's range tests, $p>0.1$ ).

Table 7. Summary of four-way ANOVA comparing associations between HC-SSs and NC-GEs ${ }^{a}$

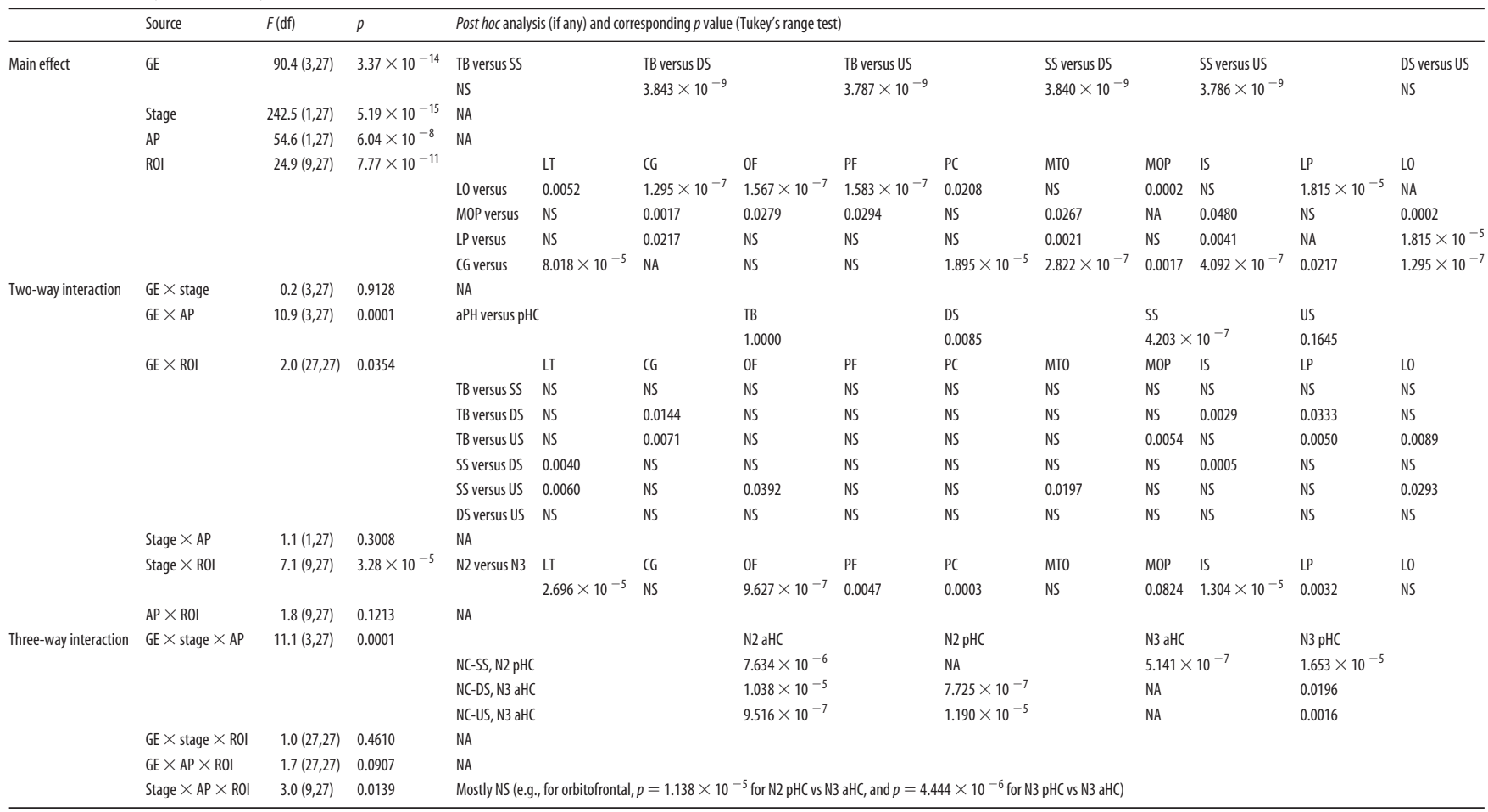

${ }^{a}$ For each HC-NC electrode pair and sleep stage (N2 vs N3), the significance of the NC-GE occurrence times relative to the HC-SS onset was determined (see Fig. $3 B$; Materials and Methods). We then tallied the proportion of significant channe pairs according to their HC location (aHC, pHC), NC location (see Extended Data Table 4-1; Fig. 5A), and GE type (TB, DS, SS, US). These proportions were then entered into ANOVA. L0, Lateral occipital; LT, lateral temporal; (G, cingulate; OF, orbitofrontal; PF, prefrontal; PC, paracentral; MT0, medial temporo-occipital; MOP, medial occipito-parietal; IS, insula; LP, lateral parietal; NS, not significant (for post hoc Tukey's range tests, $p>0.1$ ).

regions had the weakest associations, particularly in N3 (Fig. $5 B$; Table 6).

Except for GE $\times$ ROI, all two-way interaction effects from the SSR ANOVA were significant. None of the three-way interaction effects were significant. The lack of significance for GE $\times$ ROI interaction suggests that, overall, the spatial pattern of HC-NC association does not differ across NC-GE types. The GE $\times$ NREM stage interaction was due to NC-SS interacting less with HC-SSRs than do USs and DSs, in N2 but not in N3. The GE $\times$ aHC/pHC interaction was due to the absence of a $\mathrm{pHC}$ advantage for TBs in N3. For SSs, in particular, pHC proportions were approximately twice as large as aHC proportions in N2, and approximately 3 times as large in N3 (Fig. 5D). The NREM stage $\times$ aHC/pHC interaction was due to a relatively greater difference in $\mathrm{HC}-\mathrm{NC}$ coupling by pHC versus aHC during in $\mathrm{N} 2$ than $\mathrm{N} 3$ (0.1841 vs 0.1090) (Fig. 5D). The NREM stage $\times$ NC ROI interaction was 
Table 8. Proportions of HC-NC channel pairs with significant GE-SSR or GE-HC SS relationships across NC, separated by ROI, NREM stage, NC-GE type, and ipsilateral/contralateral HC-NC pairings

\begin{tabular}{|c|c|c|c|c|c|c|c|c|c|c|c|c|c|c|c|c|c|}
\hline \multirow{4}{*}{$\begin{array}{l}\text { HC } \\
\text { event }\end{array}$} & \multirow{4}{*}{$\begin{array}{l}\text { ROI/SWR } \\
\text { source }\end{array}$} & \multicolumn{16}{|c|}{ Proportion of channels with significant NC-GE and HC-SS/SSR co-occurrence } \\
\hline & & \multicolumn{4}{|l|}{ TB } & \multicolumn{4}{|l|}{ DS } & \multicolumn{4}{|l|}{ SS } & \multicolumn{4}{|l|}{ US } \\
\hline & & \multicolumn{2}{|l|}{ N2 } & \multicolumn{2}{|l|}{ N3 } & \multicolumn{2}{|l|}{ N2 } & \multicolumn{2}{|l|}{ N3 } & \multicolumn{2}{|l|}{ N2 } & \multicolumn{2}{|l|}{ N3 } & \multicolumn{2}{|l|}{ N2 } & \multicolumn{2}{|l|}{ N3 } \\
\hline & & Ipsilateral & Contralateral & Ipsilateral & Contralateral & Ipsilateral & Contralateral & Ipsilateral & Contralateral & Ipsilateral & Contralateral & Ipsilateral & Contralateral & Ipsilateral & Contralateral & Ipsilateral & Contralateral \\
\hline \multirow[t]{2}{*}{ SSR } & Frontocentral & 0.206 & 0.133 & 0.099 & 0.133 & 0.518 & 0.467 & 0.234 & 0.333 & 0.362 & 0.200 & 0.255 & 0.200 & 0.553 & 0.467 & 0.270 & 0.200 \\
\hline & Nonfrontal & 0.169 & 0.138 & 0.052 & 0.049 & 0.436 & 0.228 & 0.225 & 0.179 & 0.300 & 0.260 & 0.169 & 0.098 & 0.495 & 0.382 & 0.228 & 0.171 \\
\hline \multirow[t]{2}{*}{ SS } & Frontocentral & 0.560 & 0.467 & 0.305 & 0.267 & 0.716 & 0.600 & 0.475 & 0.467 & 0.433 & 0.333 & 0.206 & 0.267 & 0.738 & 0.800 & 0.461 & 0.400 \\
\hline & Nonfrontal & 0.381 & 0.358 & 0.179 & 0.114 & 0.704 & 0.569 & 0.404 & 0.317 & 0.375 & 0.317 & 0.169 & 0.130 & 0.710 & 0.618 & 0.453 & 0.374 \\
\hline
\end{tabular}

due to some locations (orbitofrontal, lateral temporal, prefrontal, paracentral, and insula) showing stronger coupling during N2 than N3, whereas others (generally more posterior) were approximately equal (Fig. $5 B$ ). Finally, the $\mathrm{aHC} / \mathrm{pHC} \times \mathrm{NC}$ ROI interaction was due to a significant $\mathrm{aHC} / \mathrm{pHC}$ preference limited to cingulate, prefrontal, and medial occipitoparietal regions (Fig. $5 B)$, although both aHC-SSRs and pHC-SSRs appeared to engage the whole cortex (Fig. 3A; Fig. 4A; Extended Data Fig. 4-1).

In a companion paper (Jiang et al., 2019), we performed a four-way ANOVA equivalent to the one above, but for $\mathrm{HC}$ SWRs. To compare the previous results with SWRs to the current results with SSRs, we included both datasets in a five-way ANOVA with factors of SWRs/SSRs, NC-GE type (TB, DS, SS, US), NREM stage (N2/N3), aHC/pHC, and ROI. The normality assumption was checked ( $p=0.1478$, Lilliefors test on residuals). We found that HC-SWR/NC-GE coupling was more likely than HC-SSR/NC-GE coupling (SWR/SSR main effect, $F_{(1,27)}=$ $\left.117.4, p=2.471 \times 10^{-11}\right)$. Coupling of SWRs/SSRs to NC-GEs significantly interacted with the type of NC-GE $\left(F_{(3,27)}=51.57\right.$, $\left.p=2.621 \times 10^{-11}\right)$, the NREM stage $\left(F_{(1,27)}=7.27, p=0.0119\right)$, and whether the SWRs/SSRs were generated in aHC versus $\mathrm{pHC}$ $\left(F_{(1,27)}=4.57, p=0.0417\right)$. However, the SWR/SSR $\times$ NC ROI interaction showed a trend $\left(F_{(9,27)}=2.11, p=0.0650\right)$. Notably, post hoc tests revealed that the SWR/SSR $\times$ NC-GE interaction effect was due to HC-SSRs preferentially coupling with NC-SSs $(p=0.0013)$, whereas other NC-GE types are more strongly associated with HC-SWRs $\left(p=1.591 \times 10^{-4}\right.$ for TBs, $p=$ $6.027 \times 10^{-8}$ for DSs, $p=6.616 \times 10^{-8}$ for USs). Overall, these results are consistent with the special relationship observed between pHC-SSRs and NC-SSs.

\section{Differences in the NC-GE to HC-SS relationship across NC regions, NREM stages, $\mathrm{HC}$ sites, and NC-GE types}

We repeated the analysis described above for HC-SSRs, but for HC-SSs, and summarized the results in Table 7. For the ANOVA performed over proportions of NC channels with significant NC-GE to HC-SS temporal correlation, all four main effects were significant. The GE main effect was due to a larger proportion of responsive HC-NC channel pairs for DSs (0.5484) and USs (0.5658) than for TBs (0.3165) and SSs (0.3160) (Fig. 5E). The NREM stage main effect was due to more HC-NC coupling in N2 (0.5507) than $\mathrm{N} 3(0.3226)$. The $\mathrm{aHC} / \mathrm{pHC}$ main effect was due to more HC-NC coupling by pHC (0.4907) than aHC (0.3826), consistent with our previous observation of higher HC-SS density in pHC than in aHC (Fig. $1 E$; Extended Data Fig. 1-1A, B). All the above effects were comparable to their counterparts in the previous ANOVA regarding HC-SSRs, with the exception that NC-SSs were significantly more related to HC-SSRs than NC-TBs were, but no such significant difference was observed for HC-SSs overall. Thus, HC-SSRs could involve a subset of HC-SSs with preferential coupling to NC-SSs. The ROI main effect was due to greater coupling by $\mathrm{NC}$ regions, such as the cingulate, versus low levels by other regions (e.g., lateral occipital) (Fig. 5C). These results and post hoc analysis agree with their counterparts from the HC-SSR ANOVA.

The $\mathrm{GE} \times \mathrm{aHC} / \mathrm{pHC}$ interaction was due to aHC/pHC differences being confined to DSs and SSs. For NC-SSs, in particular, pHC proportions were approximately twice as large as aHC proportions in N2, and more than twice as large in N3 (Fig. $5 E$ ). The $\mathrm{GE} \times \mathrm{ROI}$ interaction was due to a greater coupling of certain NC-GE types in particular ROIs. The insula, for instance, had higher coupling to DSs (0.5833) and USs (0.3485) than TBs $(0.2197)$ and SSs (0.1742). The NREM stage $\times$ NC ROI interaction was a larger coupling in N2 than N3, especially in orbitofrontal and insula ROIs, compared with the small N2/N3 difference in, for example, cingulate and lateral occipital (Fig. 5C). Notably, for both HC-SSR and HC-SS ANOVAs, all ROIs involving occipital cortices (medial temporo-occipital, medial occipitoparietal, and lateral occipital) showed consistent associations with $\mathrm{HC}$ across $\mathrm{N} 2$ and N3, whereas orbitofrontal, prefrontal, paracentral, lateral temporal, and insula ROIs showed preferential associations in $\mathrm{N} 2$.

The GE $\times$ NREM stage $\times$ aHC/pHC interaction is presented in Figure 5E, where, for example, N2 pHC dominated for NC-SS relations. The NREM stage $\times \mathrm{aHC} / \mathrm{pHC} \times \mathrm{NCROI}$ interaction is illustrated in Figure $5 C$, where N2 pHC proportion was dominant in some ROIs (e.g., orbitofrontal), whereas others appeared more balanced (e.g., cingulate, lateral occipital). Overall, with minor exceptions, the NC-GE coupling pattern with HC-SS is similar to that of HC-SSRs.

Relationships between NC-GEs and HC-SS/SSRs in ipsilateral versus contralateral HC-NC channel pairs

Given the well-known lateralization of human hippocampal function (Glosser et al., 1998), we were interested in examining whether ipsilateral HC-NC channel pairs would show different relationships from contralateral pairs across NC-GE types, NREM stages, or NC ROIs. Due to a sparse representation of contralateral channel pairs for individual ROIs, we combined the 10 NC ROIs used in previous analysis into two: "frontocentral," which included the previous cingulate, orbitofrontal, prefrontal, and paracentral ROIs; and "nonfrontal," which included the previous lateral temporal, medial temporo-occipital, medial occipitoparietal, insula, lateral occipital, and lateral parietal ROIs. We then computed for each ROI the proportion of NC channels significantly coupled to HC-SSRs/HC-SSs (Table 8) and tallied the numbers of NC channels with or without significant HC-NC associations for each NC-GE type across N2 and N3 (Table 9).

We checked the normality assumption for ANOVA by conducting the Lilliefors test on the residuals; while the null hypothesis of normality could not be rejected for HC-SSs $(p=0.5000)$, the null hypothesis was rejected for SSRs $(p=0.0030)$. Never- 
Table 9. Numbers and proportions of ipsilateral and contralateral NC channels showing significant HC-NC GE coupling ${ }^{a}$

\begin{tabular}{|c|c|c|c|c|c|c|c|c|c|c|c|c|c|}
\hline \multirow[b]{3}{*}{$\begin{array}{l}\mathrm{HC} \\
\text { event }\end{array}$} & \multirow{3}{*}{$\begin{array}{l}\text { Stage } \\
\text { (aHC and pHC } \\
\text { combined) } \\
\text { NC-GE } \\
\text { type }\end{array}$} & \multicolumn{6}{|l|}{$\mathrm{N} 2$} & \multicolumn{6}{|l|}{ N3 } \\
\hline & & \multicolumn{3}{|l|}{ Ipsilateral } & \multicolumn{3}{|c|}{ Contralateral } & \multicolumn{3}{|l|}{ Ipsilateral } & \multicolumn{3}{|c|}{ Contralateral } \\
\hline & & $\begin{array}{l}\text { No. of } \\
\text { significant } \\
\text { channels }\end{array}$ & $\begin{array}{l}\text { No. of } \\
\text { nonsignificant } \\
\text { channels }\end{array}$ & Proportion & $\begin{array}{l}\text { No. of } \\
\text { significant } \\
\text { channels }\end{array}$ & $\begin{array}{l}\text { No. of } \\
\text { nonsignificant } \\
\text { channels }\end{array}$ & Proportion & $\begin{array}{l}\text { No. of } \\
\text { significant } \\
\text { channels }\end{array}$ & $\begin{array}{l}\text { No. of } \\
\text { nonsignificant } \\
\text { channels }\end{array}$ & Proportion & $\begin{array}{l}\text { No. of } \\
\text { significant } \\
\text { channels }\end{array}$ & $\begin{array}{l}\text { No. of } \\
\text { nonsignificant } \\
\text { channels }\end{array}$ & Proportion \\
\hline \multirow[t]{4}{*}{ SSR } & TB & 81 & 367 & 0.1808 & 19 & 119 & 0.1377 & 30 & 418 & 0.0670 & 8 & 130 & 0.0580 \\
\hline & DS & 207 & 241 & 0.4621 & 35 & 103 & 0.2536 & 102 & 346 & 0.2277 & 27 & 111 & 0.1957 \\
\hline & SS & 143 & 305 & 0.3192 & 35 & 103 & 0.2536 & 88 & 360 & 0.1964 & 15 & 123 & 0.1087 \\
\hline & US & 230 & 218 & 0.5134 & 54 & 84 & 0.3913 & 108 & 340 & 0.2411 & 24 & 114 & 0.1739 \\
\hline \multicolumn{2}{|c|}{ Mean over NC-GE types } & 165 & 283 & 0.3689 & 36 & 102 & 0.2591 & 82 & 366 & 0.1831 & 19 & 120 & 0.1341 \\
\hline \multirow[t]{4}{*}{ SS } & TB & 196 & 252 & 0.4375 & 51 & 87 & 0.3696 & 98 & 350 & 0.2188 & 18 & 120 & 0.1304 \\
\hline & DS & 317 & 131 & 0.7076 & 79 & 59 & 0.5725 & 191 & 257 & 0.4263 & 46 & 92 & 0.3333 \\
\hline & SS & 176 & 272 & 0.3929 & 44 & 94 & 0.3188 & 81 & 367 & 0.1808 & 20 & 118 & 0.1449 \\
\hline & US & 322 & 126 & 0.7188 & 88 & 50 & 0.6377 & 204 & 244 & 0.4553 & 52 & 86 & 0.3768 \\
\hline \multicolumn{2}{|c|}{ Mean over NC-GE types } & 253 & 195 & 0.5642 & 66 & 73 & 0.4747 & 144 & 305 & 0.3198 & 34 & 104 & 0.2464 \\
\hline
\end{tabular}

${ }^{a}$ Overall, across all GEs, the proportions of significant channels were lower for contralateral than for ipsilateral.

theless, we could observe in Table 9 consistent reduction in the proportions of NC channels showing significant temporal correlation with HC-SSRs, by $13 \%-45 \%$ for each NC-GE type (separately in $\mathrm{N} 2$ or N3) from ipsilateral to contralateral, with $\mathrm{NC}-\mathrm{TB}$ in N3 showing the least drop and DS in N2 showing the most. Similarly, for HC-SSs, we observed a consistent reduction across NC-GE types in both $\mathrm{N} 2$ and N3; with the exception of NC-TBs in $\mathrm{N} 3$ (40\% drop for HC-SSs vs $13 \%$ for HC-SSRs), the ipsilateral-to-contralateral drop $(11 \%-20 \%)$ in HC-NC association proportions was smaller with respect to HC-SSs than to HC-SSRs. This suggests that HC-NC communication for spindles preferentially takes place ipsilaterally, and our subsequent PAC analyses appeared to support this (Fig. 6), as we would describe in detail later in Results.

We performed four-way ANOVA as previously described for NC-GE to HC-SS associations, with the ipsilateral/contralateral factor replacing the $\mathrm{aHC} / \mathrm{pHC}$ factor. All post hoc analyses were performed with Tukey's range test. All four main effects were significant, but none of the interaction effects was significant. As our previous ANOVA already revealed significant main effects for NC-GE type and NREM stage, we focused our attention on the remaining two factors (i.e., ipsilateral/contralateral and combined NC ROI), which provided results beyond those explored in the previous ANOVAs. The main effect of ipsilateral/contralateral channel pair types yielded $F=12.55, p=0.0383$ and, together with mean proportions of 0.454 for ipsilateral and 0.400 for contralateral, suggested that more of $\mathrm{NC}$ were related to ipsilateral HC-SSs than to contralateral HC-SSs, although the difference was modest. The main effect of ROIs yielded $F=28.90, p=$ 0.0126 . Since only two ROIs were included in this ANOVA, this result suggested that the more frontal/cingulate parts of $\mathrm{NC}$ were significantly more likely to show significant associations between NC-GEs and HC-SSs than the posterior regions (mean proportion of 0.468 for frontocentral vs 0.386 for nonfrontal).

\section{HC-SSs phase-lock to NC-SSs, and NC-SSs modulate HC ripple activity}

A previous report of human HC-SSs suggested that scalp SSs could phase-lock with HC-SSs, which modulated ripple activity (Staresina et al., 2015). We observed earlier in the results that hippocampal ripples could indeed co-occur with and appeared modulated by HC-SSs (Fig. 2C,D), and we found widespread NC channels showing significant HC-NC SS temporal correlations, particularly in $\mathrm{N} 2$ with respect to $\mathrm{pHC}$ (Figs. 4B, 5E). To further evaluate spindle-mediated HC-NC coupling, we computed PLVs over $3 \mathrm{~s}$ trials where NC-SSs overlapped with HC-SSs for at least one cycle (for examples, see Fig. 6A). After PLV computation, for each nonoverlapping $50 \mathrm{~ms}$ time bin, a two-sample $t$ test was performed between the actual PLV and the baseline estimate, with the resulting $p$ values undergoing FDR correction. A given $\mathrm{HC}-\mathrm{NC}$ channel pair would be considered significantly phaselocking if: (1) >40 trials were used in the PLV computation, since small sample size is known to introduce bias (Aydore et al., 2013); or (2) at least 3 consecutive time bins yield post-FDR $p$ values $<0.05$. Since HC-SS modulates ripple activity, NC-SS phaselocking with HC-SS would modulate $\mathrm{HC}$ ripples by inference; we nonetheless also performed PAC analysis between NC-SS phase and $\mathrm{HC}$ ripple amplitude in $\mathrm{HC}-\mathrm{NC}$ channel pairs with significant SS phase-locking (for examples, see Fig. $6 B$ ), and estimated the significance of observed PAC by computing average Modulation Indices across trials (each with the same HC-NC SS overlap criterion as used for PLV) and comparing them with bootstrapped baselines (Tort et al., 2010).

We found that significant HC-NC SS phase-locking (over $10-16 \mathrm{~Hz}$, with maximum PLV ranging from 0.3 to 0.9 ) could be found across widespread NC regions (Fig. 6C; Extended Data Fig. 6-1). Maximum PLV tended to be reached at $\sim 300-700 \mathrm{~ms}$ after HC-SS onsets. We observed a higher density of NC channels showing significant PLV with $\mathrm{pHC}$ than with aHC. In particular, $37 \%$ of NC channels phase-locked with $\mathrm{HC}$; and out of this channel population, $67 \%$ phase-locked with $\mathrm{pHC}$ (vs $33 \%$ with aHC) in $\mathrm{N} 2$, and $70 \%$ phase-locked with pHC (vs 30\% with aHC) in $\mathrm{N} 3$. For both $\mathrm{N} 2$ and N3, this pHC preference among NC channels with significant SS PLV was significant (Fisher's exact tests, $p=4.991 \times 10^{-9}$ for N2, $p=2.377 \times 10^{-7}$ for N3). While HC-NC SS phase-locking was observed across NC, only a small proportion had sustained large PLV (e.g., Fig. 6A2,A4). Specifically, only $5 \%$ of all NC channels had a peak HC-NC SS PLV value $>0.4$; in this small population of high PLV channels, $\sim 70 \%$ were parietal ( 7 in precuneus, 5 in angular gyrus, 4 in intraparietal sulcus). In addition, for patients with both aHC and pHC contacts in the same hippocampus, NC channels phase-locked to $\mathrm{pHC}$ tended not to show significant phase-locking with aHC as well (e.g., Fig. 6A), suggesting that $\mathrm{HC}-\mathrm{NC}$ coupling mediated by spindle phase could be strongly localized along the HC longitudinal axis, which appears in agreement with the rare overlap be- 
A Time course of SS phase-locking value (PLV) between $\mathrm{HC}$ and $\mathrm{NC}$

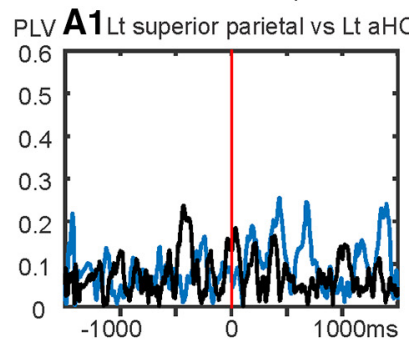

PLV A3 Rt precuneus vs Lt pHC

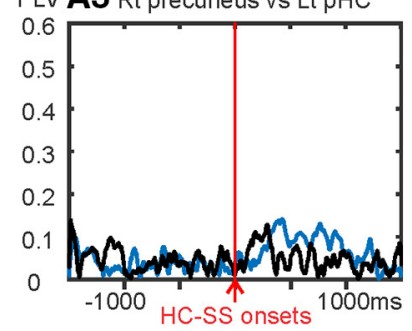

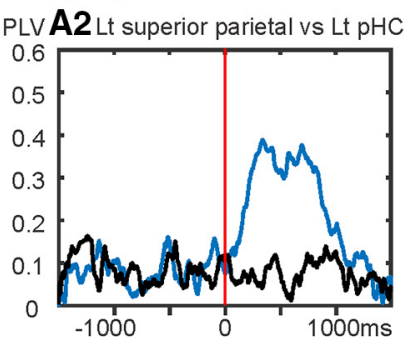

PLV A4 Rt precuneus vs Rt $\mathrm{pHC}$

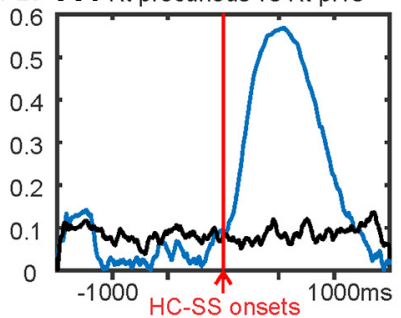

B Phase-amplitude coupling of $\mathrm{pHC}$-SSR to parietal SS

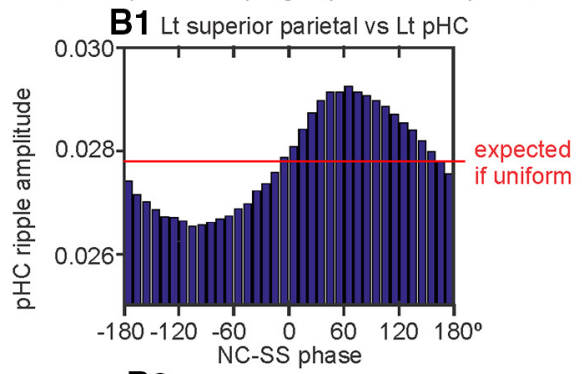

B2 Rt precuneus vs Rt pHC

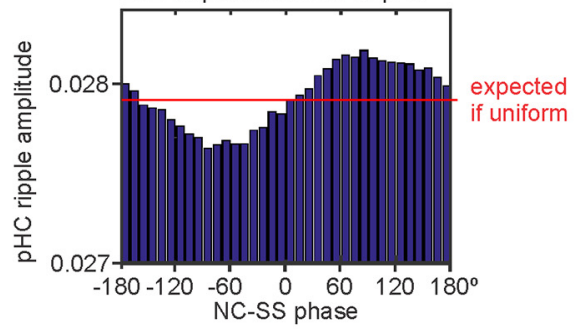

C Cortical distribution of SS phase-locking with $\mathrm{HC}$

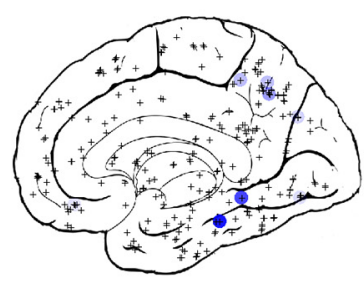

$\mathrm{N} 2, \mathrm{aHC}$

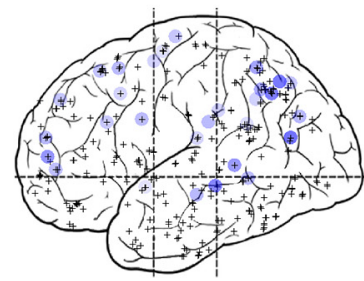

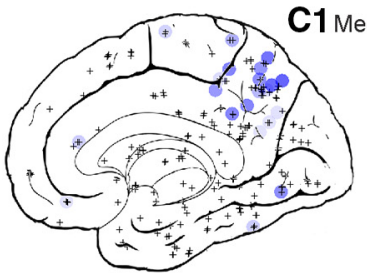

$\mathrm{N} 2, \mathrm{pHC}$

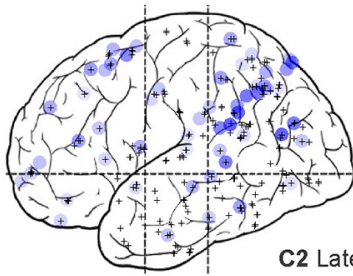

C2 Lateral NC

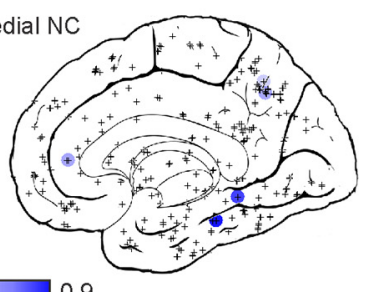

$\mathrm{N} 3, \mathrm{aHC}$

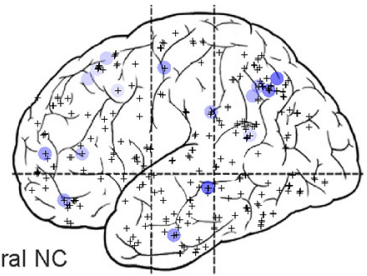

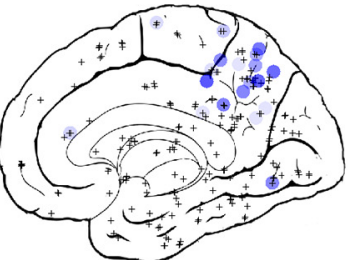

N3, pHC

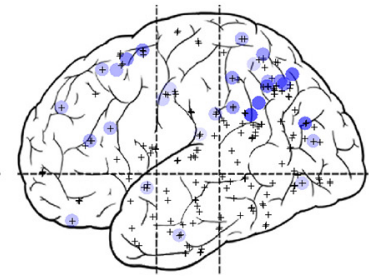

Figure 6. Parietal spindles phase-lock to hippocampal spindles and modulate hippocampal ripple activity. $\boldsymbol{A}, \mathrm{HC}-\mathrm{SS}$ phase-locking with NC-SS tends to be localized along the longitudinal axis ( $\boldsymbol{A} 1$ vs $\mathbf{A 2}$ ) and prefers ipsilateral HC-NC channel pairs ( $\mathbf{A} 3$ vs $\boldsymbol{A}$ 4). Each panel shows two traces of PLV. Blue trace represents average PLV computed over 3 s trials with overlapping HC-SSs and NC-SSS, centered on the starts of HC-SSs. Black trace represents average PLV computed over an equal number of pseudo-trials centered on random time points in NREM (N2 + N3). Lt, Left; Rt, right. $\boldsymbol{B}$, PAC between the phase of NC-SS $(10-16 \mathrm{~Hz})$ in parietal lobe and the amplitude of HC ripple-range activity $(60-120 \mathrm{~Hz})$. Each histogram bin covers 10 degrees of phase. $\boldsymbol{B} \mathbf{1}, \mathbf{B} \mathbf{2}$, Channels are the same as those in $\mathbf{A} \mathbf{2}$ and $\boldsymbol{A 4}$, respectively. C, $2 \mathrm{D}$ projection maps of medial (C) and lateral (C2) NC channels showing significant spindle phase-locking with HC. Each blue circle represents a significant NC channel, with color intensity corresponding to peak PLV amplitude. Plus signs indicate channels with no significant phase-locking. Projection maps that separate left and right hemispheres can be found in Extended Data Figure 6-1.

tween aHC- and pHC-SSRs/SSs reported in the beginning of Results.

Similar to the result above with high PLV channel pairs being a small subset of all channels with significant PLV, NC-SS phase to $\mathrm{HC}$ ripple amplitude $\mathrm{PAC}$ was only observed in approximately one-fourth of all channels with HC-NC-SS phase-locking. Again, these channels were mainly ( $\sim 70 \%$ ) parietal ( 6 in precuneus, 3 in angular gyrus, 3 in intraparietal sulcus). Interestingly, only half of the high SS PLV channel pairs showed significant NC-SS to HC ripple PAC also.

Remarkably, for the population of NC channels from the 8 patients with multiple $\mathrm{HC}$ contacts in the same hippocampi, 15 of the $16(94 \%)$ NC channels showing significant SS phase-locking with aHC also phase-lock with pHC significantly in N2, whereas conversely only 15 of the 43 (35\%) NC channels showing signif- icant SS phase-locking with pHC also phase-lock with aHC; in $\mathrm{N} 3$, this aHC/pHC imbalance in percentages of concurrently coupled NC sites was preserved ( 7 of 10, 70\% with aHC vs 7 of 24 , $29 \%$ with pHC). Thus, if aHC phase-locks SS with an NC site, then $\mathrm{pHC}$ does also, but not vice versa.

Strong ipsilateral preference for phase-locked HC-NC channel pairs was also observed in the patients with bilateral $\mathrm{pHC}$ contacts, with example PLV plots shown in Figure $6 A$, where the right precuneus preferentially coupled with right $\mathrm{pHC}$. Within the channel pairs showing significant SS PLV involving aHC, in $\mathrm{N} 2$, only $5 \%$ were contralateral (thus $95 \%$ were ipsilateral); in N3, all of the channel pairs with significant PLV involving aHC were ipsilateral. Similarly, for phase-locking channel pairs involving pHC, $89 \%$ were ipsilateral in $\mathrm{N} 2$ and $90 \%$ were ipsilateral in N3. This strong preference for ipsilateral HC-NC SS phase-locking is 


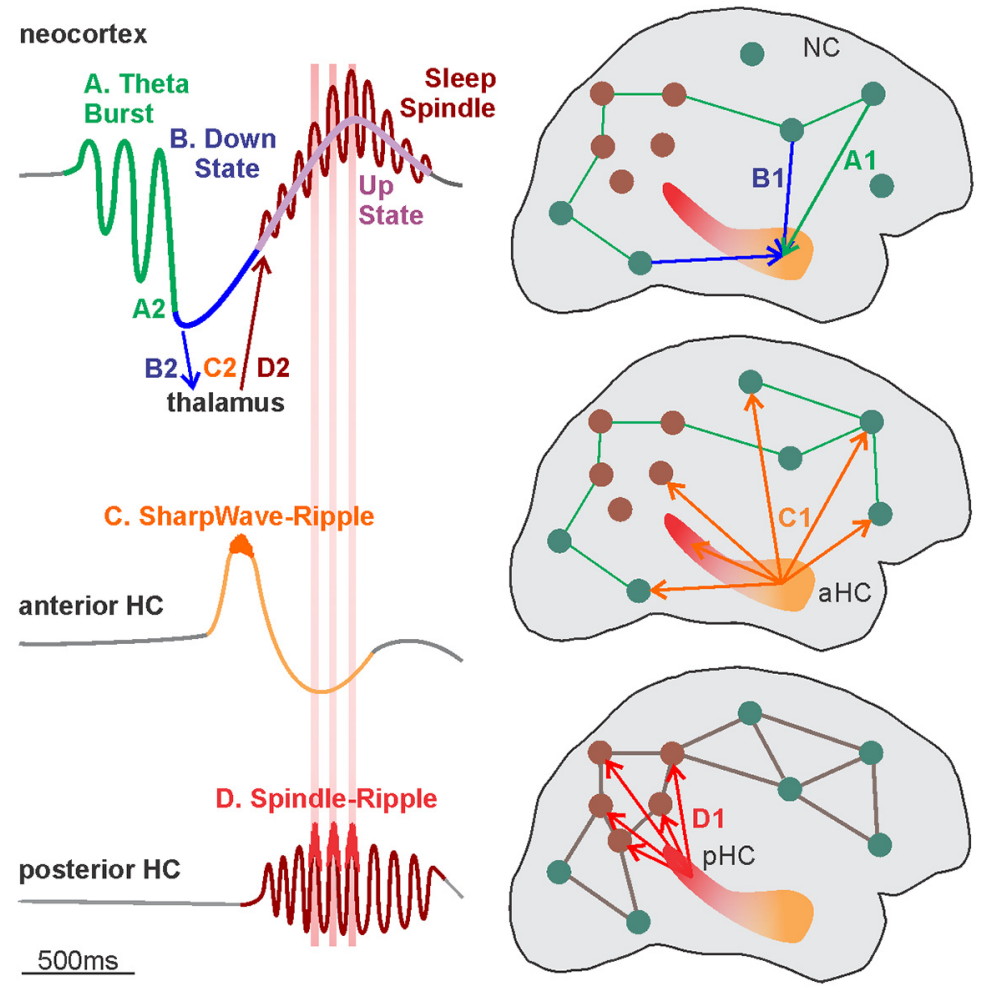

Figure 7. Proposed HC-SWR/SSR interactions with NC-GEs in the context of previous findings of NC-HC-thalamic interactions during GEs (Mak-McCully et al., 2017; Gonzalez et al., 2018; Jiang et al., 2019). $\boldsymbol{A}$, The sequence begins with a spontaneous NC-TB. Firing patterns are hypothesized to project to the $\mathrm{HC}$ and help inform the context in which they arise (A1). Locally, the NC-TB may lead to the NC-DS (A2). $\boldsymbol{B}$, The NC-DS in turn may contribute to the broadband decrease in HC-LFP preceding SWR (B1) and trigger a thalamic-DS (B2). C, The HC-SWR occurs at this critical juncture (Jiang et al., 2019), contributing recent memory traces to the NC as it reboots from the NC-DS (C1); meanwhile, the thalamic-DS enables the h and T currents to generate a thalamic-SS (C2). D, The pHC-SSR follows, perhaps projecting more detailed recent memory information during multiple ripples (D1). The pHC-SSRs are tightly phase-locked to SS in inferior and medial parietal cortices (present study), projected from the thalamus (D2). Increased firing and $\mathrm{Ca}^{2+}$ influx during the NC-SS and underlying NC-US may provide an ideal cellular environment for memory consolidation. Experimental evidence is lacking for pHC-SSRs serving as markers for replay, and the evidence for aHC-SWRs is mainly from rodents.

larger than the preference for ipsilateral co-occurrence of HC-SSs and HC-SSRs with NC-GEs described above.

\section{Discussion}

This study quantifies the characteristics of ripples that co-occur with hippocampal spindles (HC-SSRs) in humans. We previously demonstrated that human SWRs (HC-SWRs) resemble animal HC-SWRs in their density, NREM concentration, waveform, and intrahippocampal topography (Jiang et al., 2019). HCSSRs and HC-SWRs were distinct in important ways: HC-SSRs were dominant in $\mathrm{pHC}$, whereas HC-SWRs were in aHC. Both HC-SSRs and HC-SWRs were coupled with all types of cortical GEs (TBs, DSs, USs, and SSs) in widespread bilateral locations (Fig. 7). However, HC-SSRs were tightly phase-locked with SSs in ipsilateral inferior parietal and precuneate sites, whereas HCSWRs showed a preference for prefrontal, central, and cingulate cortices. HC-SSRs generally occurred during the NC-SSs/USs, whereas HC-SWRs generally occurred $\sim 400 \mathrm{~ms}$ earlier, during the NC-DSs. A single HC-SSR is commonly adjacent to multiple ripples on successive peaks of the same HC-SSs, separated by $\geq 50$ ms, whereas single ripples on HC-SWRs were separated by $\sim 5 \mathrm{~s}$. These differences suggest that HC-SSRs and HC-SWRs may potentially play complementary roles in memory consolidation, although further support from additional behavioral studies is needed, as our current study lacks behavioral assess- ments that could clearly demonstrate the involvement of HC-SS/SSR in memory consolidation.

Human HC-SSs have previously been recorded in NREM (Montplaisir et al., 1981; Malow et al., 1999; Andrillon et al., 2011; Carpentier et al., 2017) and REM sleep (Lestra et al., 2018). We observed SSs in $\mathrm{HC}$ with no ictal or interictal involvement, and HC-SSs are observed in animals (Miyawaki and Diba, 2016), suggesting that they represent healthy activity. Our confirmation of phase modulation of $\mathrm{HC}$ ripples by HC-SSs, and our demonstration of their coordination with NC-GEs provides a possible framework for HC-NC information transfer, as previously suggested (Siapas and Wilson, 1998; Sirota et al., 2003; Staresina et al., 2015) Antony et al., 2018). However, despite our efforts to eliminate contamination by epileptiform events, our study is limited by the possibility of pathological restructuring, and the possible role of SSRs in memory replay is unconfirmed in mechanistic studies.

Animal studies have described varying temporal relationships between HCSWRs and NC-SSs. The possibility that NC-SSs play an initiating role is implied by the observation that SSs can reach the hippocampus via entorhinal cortex and phase-modulate HC firing (Sirota et al., 2003; Isomura et al., 2006). However, others found HC-SWRs to precede NC-SSs (Siapas and Wilson, 1998; Peyrache et al., 2011). This apparent discrepancy could reflect the fact that, while HC-SWRs tend to precede NC-SSs (Jiang et al., 2019), HC-SSRs tend to co-occur with NC-SSs (Fig. 4A; Extended Data Fig. 4-1). Since some of the reports cited above detected highfrequency power without verifying a co-occurring sharpwave waveform, it is possible that they studied a mixed population of SWRs and SSRs.

Clemens et al. (2011) reported that ripples (including both interictal spikes and physiological activity) recorded medially to the parahippocampal gyrus are phase-modulated by parietal scalp spindles. Similarly, Staresina et al. (2015) found a 5\% increase in HC ripple band power $\pm 250 \mathrm{~ms}$ to scalp spindles. However, the current report appears to provide the first evidence that human intracranially recorded NC-SS phase-lock with HC-SS and show PAC with ripples. HC and parietal spindles may become phase-locked via projections from CA1 to area 7a (Clower et al., 2001), thalamohippocampal (Amaral and Cowan, 1980), and/or thalamocortical projections (Mak-McCully et al., 2017) (Fig. 7B-D).

An important consequence of this co-occurrence and phaselocking of HC-SWRs with NC-SSs is that, if HC-SWRs mark replay firing, then this information would be projected to $\mathrm{NC}$ at a propitious time (Fig. 7D). In humans, NC-SSs (Hagler et al., 2018), and especially NC-SSs/USs, increase firing (Csercsa et al., 2010; Mak-McCully et al., 2017; Gonzalez et al., 2018). In rodents, NC-SSs (Seibt et al., 2017), and especially NC-SSs/USs (Niethard et al., 2018), are associated with strong $\mathrm{Ca}^{2+}$ influx in 
NC pyramidal cell apical dendrites, and increased plasticity (Steriade and Timofeev, 2003). Behavioral consolidation in rodents depends on NC-SS/US coupling with HC-SWRs (Maingret et al., 2016; Latchoumane et al., 2017). Similarly, scalp SS and SS-US complexes are associated with consolidation in humans (Marshall et al., 2006; Mednick et al., 2013; Niknazar et al., 2015).

The distinctive behavioral contributions of ventral/dorsal HC in rodents, or aHC/pHC in humans, have been characterized in multiple ways. In rodents, ventral HC lesions impair contextual emotional learning, corresponding to its amygdala, orbitofrontal, and hypothalamic connections, whereas dorsal lesions impair spatial learning, corresponding to its retrosplenial and parietal connections (Fanselow and Dong, 2010). Although ventral HC neurons also exhibit place fields, they are less frequent and larger than dorsal HC (Strange et al., 2014), and the smaller ventral HC dentate gyrus may support coarser pattern separation (Poppenk et al., 2013). The anatomical contrasts between ventral and dorsal $\mathrm{HC}$ in rodents have been confirmed with resting-state BOLD connectivity measures in humans (Ranganath and Ritchey, 2012). Functional imaging studies suggest greater aHC involvement in learning the gist of events, and pHC the details, perhaps consistent with the broader encoding by its cells in rodents (Poppenk et al., 2013; Strange et al., 2014).

The preponderance of SSRs in dorsal HC has not been reported in rodents. Indeed, SWRs are more dense and stronger in dorsal than ventral rodent HC (Patel et al., 2013). One possible interpretation is that $\mathrm{pHC}$-SSRs may support a faculty that is particularly well developed in humans. The cortical areas (angular gyrus, precuneus, and retrosplenial cortex) strongly phaselocked with pHC-SSR, as well as the HC itself, are all associated with episodic memory, which Tulving and Markowitsch (1998) have argued is unique to humans. Lesions restricted to the HC but sparing adjoining temporal cortical areas severely impair episodic memory with relatively preserved nonepisodic declarative memories (Vargha-Khadem et al., 1997). Similarly, angular gyrus lesions impair recollective experience without necessarily impairing familiarity judgments (Papagno, 2018). Recollective experience is correlated with hemodynamic activation in these areas (Gilmore et al., 2015), as well as unit increases (Rutishauser et al., 2018), possibly indicating a role in storing details of episodic memories (Rugg and King, 2018; but see Davis et al., 2018). Recollection is also associated with a parietal positive event-related potential, which is dependent on HC integrity (Smith and Halgren, 1989), and correlated across trials with $\mathrm{pHC}$ and retrosplenial BOLD activation (Hoppstädter et al., 2015). HC stimulation can induce intense feelings that current experience is being relived (déjà $v u$ ) as well as vivid recollections of previous experiences (Halgren et al., 1978; Curot et al., 2017). These considerations raise the possibility that pHC-SSRs are more prominent in humans because they help support autobiographical memory; further investigations with behavioral assessments are necessary to test this hypothesis.

The ratio of hippocampal to neocortical pyramidal cells is $\sim 1: 33$ in rats and $\sim 1: 2246$ in humans (Korbo et al., 1990; West and Gundersen, 1990; West et al., 1991; Pakkenberg and Gundersen, 1997). Thus, the $\mathrm{HC} \rightarrow \mathrm{NC}$ fanout is extremely broad in humans. Yet, a central feature of HC-dependent memory is its ability to unify arbitrary elements, and this is presumed to require the $\mathrm{HC}$ to interact with all or most of the NC (McNaughton, 2010). Indeed, we have shown that the HC-SWRs co-occur with NC-SSs/USs across widespread cortical areas in all lobes (Jiang et al., 2019). Furthermore, HC-SWRs only occur $\sim 12$ times per minute; thus, most cortical activity would develop in the absence of $\mathrm{HC}$ input from any particular location. Nonetheless, HC-SWRs tend to occur at the time of the NC-DSs, that is, at the beginning of the NC-SSs/USs (Fig. 7B), and thus can set the stage for subsequent elaboration of the $\mathrm{NC} \leftrightarrow$ $\mathrm{NC}$ network. Therefore, the wide $\mathrm{HC} \rightarrow \mathrm{NC}$ fanout and low $\mathrm{HC}$ SWR density, as well as its timing, might be expected to limit the influence of $\mathrm{HC}$ input to setting the general context of NC network evolution rather than its precise content (Fig. 7C).

In contrast to aHC-SWRs, pHC-SSRs produce multiple ripple bursts and thus replay patterns to a focal cortical target during the process of NC cell assembly. Multiple ripples commonly occur over the course of a single pHC-SSR, with their centers separated by $\geq 50 \mathrm{~ms}$, compared with HC-SWRs, which are separated by $\sim 5 \mathrm{~s}$. Furthermore, in contrast to the NC-GE co-occurrence of HC-SWRs with widespread NC areas, pHC-SSRs restrict their very high level of phase-locking during putative replay to a focal target in area 7a. Finally, the pHC-SSR input to NC is sustained during the SSs/USs when cell-firing dendritic calcium levels are optimal for synapse formation, as opposed to a single punctate input from HC-SWRs at the beginning of the SSs/USs. Thus, while the HC-SWR input appears well suited to provide guiding input to widespread NC areas comprising the "gist" of a recent event, the pHC-SSRs may be better-suited to impress specific details through repeated inputs to focal $\mathrm{NC}$ areas (Fig. 7C,D).

In conclusion, during NREM sleep, the human hippocampus generates ripples and may potentially provide specific information to the neocortex in two distinct LFP and anatomical contexts: aHC-SWR and pHC-SSR. Both are coordinated with thalamocortical waves, which occur in a typical order $\mathrm{TBs} \rightarrow$ $\mathrm{DSs} \rightarrow \mathrm{SSs} / \mathrm{USs}$, but aHC-SWRs occur with the DSs and $\mathrm{pHC}$ SSRs with SSs/USs (Fig. 7). aHC is anatomically connected with limbic and prefrontal sites, and aHC-SWRs are preferentially coupled with prefrontal and cingulate NC-GEs (Jiang et al., 2019). pHC is connected to parahippocampal, retrosplenial, precuneus, and inferior parietal cortices, and pHC-SSRs are strongly phase-locked with these areas. The low rate of aHC-SWRs, their broad bilateral anatomical fanout, and the preference of this fanout for frontolimbic sites are consistent with the contextual contribution sometimes assigned to aHC (or ventral hippocampus in rodents). In contrast, the rapid repeated ripples during pHC-SSRs, phase-locked with focal ipsilateral parietal loci, are consistent with a more detail-oriented consolidation process if, indeed, pHC-SSRs are found to participate in replay.

\section{References}

Amaral DG, Cowan WM (1980) Subcortical afferents to the hippocampal formation in the monkey. J Comp Neurol 189:573-591.

Andrillon T, Nir Y, Staba RJ, Ferrarelli F, Cirelli C, Tononi G, Fried I (2011) Sleep spindles in humans: insights from intracranial EEG and unit recordings. J Neurosci 31:17821-17834.

Antony JW, Piloto L, Wang M, Pacheco P, Norman KA, Paller KA (2018) Sleep spindle refractoriness segregates periods of memory reactivation. Curr Biol 28:1736-1743.e4.

Axmacher N, Elger CE, Fell J (2008) Ripples in the medial temporal lobe are relevant for human memory consolidation. Brain J Neurol 131: 1806-1817

Aydore S, Pantazis D, Leahy RM (2013) A note on the phase locking value and its properties. Neuroimage 74:231-244.

Battaglia FP, Sutherland GR, McNaughton BL (2004) Hippocampal sharp wave bursts coincide with neocortical "up-state” transitions. Learn Mem 11:697-704

Benjamini Y, Hochberg Y (1995) Controlling the false discovery rate: a practical and powerful approach to multiple testing. J R Stat Soc Ser B Methodol 57:289-300.

Bragin A, Penttonen M, Buzsáki G (1997) Termination of epileptic afterdischarge in the hippocampus. J Neurosci 17:2567-2579.

Bragin A, Engel J Jr, Wilson CL, Fried I, Buzsáki G (1999) High-frequency oscillations in human brain. Hippocampus 9:137-142. 
Buzsáki G (2015) Hippocampal sharp wave ripple: a cognitive biomarker for episodic memory and planning. Hippocampus 25:1073-1188.

Buzsáki G, Horváth Z, Urioste R, Hetke J, Wise K (1992) High-frequency network oscillation in the hippocampus. Science 256:1025-1027.

Carpentier N, Cecchin T, Koessler L, Louis-Dorr V, Jonas J, Vignal JP, Carpentier M, Szurhaj W, Bourgin P, Maillard L (2017) Stereo-electroencephalography identifies N2 sleep and spindles in human hippocampus. Clin Neurophysiol 128:1696-1706.

Carskadon MA, Dement WC (2010) Monitoring and staging human sleep. In: Principles and practice of sleep medicine (Kryger MH, Roth T, Dement WC, eds), Ed 5, pp 16-26. St. Louis: Elsevier Saunders.

Cash SS, Halgren E, Dehghani N, Rossetti AO, Thesen T, Wang C, Devinsky O, Kuzniecky R, Doyle W, Madsen JR, Bromfield E, Eross L, Halász P, Karmos G, Csercsa R, Wittner L, Ulbert I (2009) The human K-complex represents an isolated cortical down-state. Science 324:1084-1087.

Clemens Z, Mölle M, Eross L, Jakus R, Rásonyi G, Halász P, Born J (2011) Fine-tuned coupling between human parahippocampal ripples and sleep spindles. Eur J Neurosci 33:511-520.

Clower DM, West RA, Lynch JC, Strick PL (2001) The inferior parietal lobule is the target of output from the superior colliculus, hippocampus, and cerebellum. J Neurosci 21:6283-6291.

Colgin LL, Denninger T, Fyhn M, Hafting T, Bonnevie T, Jensen O, Moser MB, Moser EI (2009) Frequency of $\gamma$ oscillations routes flow of information in the hippocampus. Nature 462:353-357.

Csercsa R, Dombovári B, Fabó D, Wittner L, Eross L, Entz L, Sólyom A, Rásonyi G, Szucs A, Kelemen A, Jakus R, Juhos V, Grand L, Magony A, Halász P, Freund TF, Maglóczky Z, Cash SS, Papp L, Karmos G, et al. (2010) Laminar analysis of slow wave activity in humans. Brain J Neurol 133:2814-2829.

Curot J, Busigny T, Valton L, Denuelle M, Vignal JP, Maillard L, Chauvel P, Pariente J, Trebuchon A, Bartolomei F, Barbeau EJ (2017) Memory scrutinized through electrical brain stimulation: a review of 80 years of experiential phenomena. Neurosci Biobehav Rev 78:161-177.

Dale AM, Fischl B, Sereno MI (1999) Cortical surface-based analysis: I. Segmentation and surface reconstruction. Neuroimage 9:179-194.

Davis SW, Wing EA, Cabeza R (2018) Contributions of the ventral parietal cortex to declarative memory. Handb Clin Neurol 151:525-553.

Delorme A, Makeig S (2004) EEGLAB: an open source toolbox for analysis of single-trial EEG dynamics including independent component analysis. J Neurosci Methods 134:9-21.

Desikan RS, Ségonne F, Fischl B, Quinn BT, Dickerson BC, Blacker D, Buckner RL, Dale AM, Maguire RP, Hyman BT, Albert MS, Killiany RJ (2006) An automated labeling system for subdividing the human cerebral cortex on MRI scans into gyral based regions of interest. Neuroimage 31: 968-980.

Destrieux C, Bourry D, Velut S (2013) Surgical anatomy of the hippocampus. Neurochirurgie 59:149-158.

Diekelmann S, Born J (2010) The memory function of sleep. Nat Rev Neurosci 11:114-126.

Ding SL, Van Hoesen GW (2015) Organization and detailed parcellation of human hippocampal head and body regions based on a combined analysis of cyto- and chemoarchitecture. J Comp Neurol 523:2233-2253.

Duvernoy H (1988) The human hippocampus: an atlas of applied anatomy. Munich: Bergmann-Verlag.

Dupré la Tour T, Tallot L, Grabot L, Doyère V, van Wassenhove V, Grenier Y, Gramfort A (2017) Non-linear auto-regressive models for crossfrequency coupling in neural time series. PLOS Comput Biol 13: e1005893.

Dykstra AR, Chan AM, Quinn BT, Zepeda R, Keller CJ, Cormier J, Madsen JR, Eskandar EN, Cash SS (2012) Individualized localization and cortical surface-based registration of intracranial electrodes. Neuroimage 59: 3563-3570.

Fanselow MS, Dong HW (2010) Are the dorsal and ventral hippocampus functionally distinct structures? Neuron 65:7-19.

Fischl B, Sereno MI, Dale AM (1999a) Cortical surface-based analysis: II: Inflation, flattening, and a surface-based coordinate system. Neuroimage 9:195-207.

Fischl B, Sereno MI, Tootell RB, Dale AM (1999b) High-resolution intersubject averaging and a coordinate system for the cortical surface. Hum Brain Mapp 8:272-284.

Fischl B, van der Kouwe A, Destrieux C, Halgren E, Ségonne F, Salat DH, Busa E, Seidman LJ, Goldstein J, Kennedy D, Caviness V, Makris N, Rosen B,
Dale AM (2004) Automatically parcellating the human cerebral cortex. Cereb Cortex 14:11-22.

Gervasoni D, Lin SC, Ribeiro S, Soares ES, Pantoja J, Nicolelis MA (2004) Global forebrain dynamics predict rat behavioral states and their transitions. J Neurosci 24:11137-11147.

Gilmore AW, Nelson SM, McDermott KB (2015) A parietal memory network revealed by multiple MRI methods. Trends Cogn Sci 19:534-543.

Girardeau G, Zugaro M (2011) Hippocampal ripples and memory consolidation. Curr Opin Neurobiol 21:452-459.

Girardeau G, Benchenane K, Wiener SI, Buzsáki G, Zugaro MB (2009) Selective suppression of hippocampal ripples impairs spatial memory. Nat Neurosci 12:1222-1223.

Glosser G, Deutsch GK, Cole LC, Corwin J, Saykin AJ (1998) Differential lateralization of memory discrimination and response bias in temporal lobe epilepsy patients. J Int Neuropsychol Soc 4:502-511.

Göldi M, van Poppel EA, Rasch B, Schreiner T (2019) Increased neuronal signatures of targeted memory reactivation during slow-wave up states. Sci Rep 9:2715.

Gonzalez CE, Mak-McCully RA, Rosen BQ, Cash SS, Chauvel PY, Bastuji H, Rey M, Halgren E (2018) Theta bursts precede, and spindles follow, cortical and thalamic downstates in human NREM sleep. J Neurosci 38: 9989-10001.

Gonzalez-Martinez J, Bulacio J, Alexopoulos A, Jehi L, Bingaman W, Najm I (2013) Stereoelectroencephalography in the "difficult to localize" refractory focal epilepsy: early experience from a North American Epilepsy Center. Epilepsia 54:323-330.

Hagler DJ Jr, Ulbert I, Wittner L, Eross L, Madsen JR, Devinsky O, Doyle W, Fabó D, Cash SS, Halgren E (2018) Heterogeneous origins of human sleep spindles in different cortical layers. J Neurosci 38:3013-3025.

Halgren E, Walter RD, Cherlow DG, Crandall PH (1978) Mental phenomena evoked by electrical stimulation of the human hippocampal formation and amygdala. Brain J Neurol 101:83-117.

Headley DB, Kanta V, Paré D (2017) Intra- and interregional cortical interactions related to sharp-wave ripples and dentate spikes. J Neurophysiol 117:556-565.

Hoppstädter M, Baeuchl C, Diener C, Flor H, Meyer P (2015) Simultaneous EEG-fMRI reveals brain networks underlying recognition memory ERP old/new effects. Neuroimage 116:112-122.

Isomura Y, Sirota A, Ozen S, Montgomery S, Mizuseki K, Henze DA, Buzsáki G (2006) Integration and segregation of activity in entorhinal-hippocampal subregions by neocortical slow oscillations. Neuron 52:871-882.

Jiang X, Shamie I, Doyle W, Friedman D, Dugan P, Devinsky O, Eskandar E, Cash SS, Thesen T, Halgren E (2017) Replay of large-scale spatiotemporal patterns from waking during subsequent NREM sleep in human cortex. Sci Rep 7:17380.

Jiang X, Gonzalez-Martinez J, Halgren E (2019) Coordination of human hippocampal sharpwave ripples during NREM sleep with cortical theta bursts, spindles, downstates and upstates. Advance online publication. Retrieved Sep 17, 2019. doi: 10.1523/JNEUROSCI.2857-18.2019.

Korbo L, Pakkenberg B, Ladefoged O, Gundersen HJ, Arlien-Søborg P, Pakkenberg H (1990) An efficient method for estimating the total number of neurons in rat brain cortex. J Neurosci Methods 31:93-100.

Lachaux JP, Rodriguez E, Martinerie J, Varela FJ (1999) Measuring phase synchrony in brain signals. Hum Brain Mapp 8:194-208.

Latchoumane CV, Ngo HV, Born J, Shin HS (2017) Thalamic spindles promote memory formation during sleep through triple phase-locking of cortical, thalamic, and hippocampal rhythms. Neuron 95:424-435.e6.

Lestra P, Peter-Derex L, Salin P, Comte JC (2018) Hippocampus and prefrontal cortex exhibit spindle-like waves during REM sleep. Neurophysiol Clin 48:135-136.

Maingret N, Girardeau G, Todorova R, Goutierre M, Zugaro M (2016) Hippocampo-cortical coupling mediates memory consolidation during sleep. Nat Neurosci 19:959-964.

Mak-McCully RA, Rosen BQ, Rolland M, Régis J, Bartolomei F, Rey M, Chauvel P, Cash SS, Halgren E (2015) Distribution, amplitude, incidence, co-occurrence, and propagation of human K-complexes in focal transcortical recordings. eNeuro 2:0028-15.2015.

Mak-McCully RA, Rolland M, Sargsyan A, Gonzalez C, Magnin M, Chauvel P, Rey M, Bastuji H, Halgren E (2017) Coordination of cortical and thalamic activity during non-REM sleep in humans. Nat Commun 8:15499.

Malow BA, Carney PR, Kushwaha R, Bowes RJ (1999) Hippocampal sleep 
spindles revisited: physiologic or epileptic activity? Clin Neurophysiol 110:687-693.

Marshall L, Helgadóttir H, Mölle M, Born J (2006) Boosting slow oscillations during sleep potentiates memory. Nature 444:610-613.

McNaughton BL (2010) Cortical hierarchies, sleep, and the extraction of knowledge from memory. Artif Intell 174:205-214.

Mednick SC, McDevitt EA, Walsh JK, Wamsley E, Paulus M, Kanady JC, Drummond SP (2013) The critical role of sleep spindles in hippocampal-dependent memory: a pharmacology study. J Neurosci 33:4494-4504.

Miyawaki H, Diba K (2016) Regulation of hippocampal firing by network oscillations during sleep. Curr Biol 26:893-902.

Mölle M, Yeshenko O, Marshall L, Sara SJ, Born J (2006) Hippocampal sharp wave ripples linked to slow oscillations in rat slow-wave sleep. J Neurophysiol 96:62-70.

Montplaisir J, Leduc L, Laverdière M, Walsh J, Saint-Hilaire JM (1981) Sleep spindles in the human hippocampus: normal or epileptic activity? Sleep 4:423-428.

Moraes W, Piovezan R, Poyares D, Bittencourt LR, Santos-Silva R, Tufik S (2014) Effects of aging on sleep structure throughout adulthood: a population-based study. Sleep Med 15:401-409.

Nádasdy Z, Hirase H, Czurkó A, Csicsvari J, Buzsáki G (1999) Replay and time compression of recurring spike sequences in the hippocampus. J Neurosci 19:9497-9507.

Niethard N, Ngo HV, Ehrlich I, Born J (2018) Cortical circuit activity underlying sleep slow oscillations and spindles. Proc Natl Acad Sci U S A 115:E9220-E9229.

Niknazar M, Krishnan GP, Bazhenov M, Mednick SC (2015) Coupling of thalamocortical sleep oscillations are important for memory consolidation in humans. PLoS One 10:e0144720.

O’Neill J, Pleydell-Bouverie B, Dupret D, Csicsvari J (2010) Play it again: reactivation of waking experience and memory. Trends Neurosci 33:220-229.

Oostenveld R, Fries P, Maris E, Schoffelen JM (2011) FieldTrip: open source software for advanced analysis of MEG, EEG, and invasive electrophysiological data. Comput Intell Neurosci 2011:156869.

Pakkenberg B, Gundersen HJ (1997) Neocortical neuron number in humans: effect of sex and age. J Comp Neurol 384:312-320.

Papagno C (2018) Memory deficits. In: The parietal lobe: handbook of clinical neurology, pp 377-393. Amsterdam: Elsevier.

Patel J, Schomburg EW, Berényi A, Fujisawa S, Buzsáki G (2013) Local generation and propagation of ripples along the septotemporal axis of the hippocampus. J Neurosci 33:17029-17041.

Peyrache A, Khamassi M, Benchenane K, Wiener SI, Battaglia FP (2009) Replay of rule-learning related neural patterns in the prefrontal cortex during sleep. Nat Neurosci 12:919-926.

Peyrache A, Battaglia FP, Destexhe A (2011) Inhibition recruitment in prefrontal cortex during sleep spindles and gating of hippocampal inputs. Proc Natl Acad Sci U S A 108:17207-17212.

Poppenk J, Evensmoen HR, Moscovitch M, Nadel L (2013) Long-axis specialization of the human hippocampus. Trends Cogn Sci 17:230-240.

Ramirez-Villegas JF, Logothetis NK, Besserve M (2015) Diversity of sharpwave ripple LFP signatures reveals differentiated brain-wide dynamical events. Proc Natl Acad Sci U S A 112:E6379-E6387.

Ranganath C, Ritchey M (2012) Two cortical systems for memory-guided behaviour. Nat Rev Neurosci 13:713-726.
Rugg MD, King DR (2018) Ventral lateral parietal cortex and episodic memory retrieval. Cortex 107:238-250.

Rutishauser U, Aflalo T, Rosario ER, Pouratian N, Andersen RA (2018) Single-neuron representation of memory strength and recognition confidence in left human posterior parietal cortex. Neuron 97:209-220.e3.

Seibt J, Richard CJ, Sigl-Glöckner J, Takahashi N, Kaplan DI, Doron G, de Limoges D, Bocklisch C, Larkum ME (2017) Cortical dendritic activity correlates with spindle-rich oscillations during sleep in rodents. Nat Commun 8:684.

Siapas AG, Wilson MA (1998) Coordinated interactions between hippocampal ripples and cortical spindles during slow-wave sleep. Neuron 21:1123-1128.

Silber MH, Ancoli-Israel S, Bonnet MH, Chokroverty S, Grigg-Damberger MM, Hirshkowitz M, Kapen S, Keenan SA, Kryger MH, Penzel T, Pressman MR, Iber C (2007) The visual scoring of sleep in adults. J Clin Sleep Med 3:121-131.

Sirota A, Csicsvari J, Buhl D, Buzsáki G (2003) Communication between neocortex and hippocampus during sleep in rodents. Proc Natl Acad Sci U S A 100:2065-2069.

Skaggs WE, McNaughton BL (1996) Replay of neuronal firing sequences in rat hippocampus during sleep following spatial experience. Science 271:1870-1873.

Skaggs WE, McNaughton BL, Permenter M, Archibeque M, Vogt J, Amaral DG, Barnes CA (2007) EEG sharp waves and sparse ensemble unit activity in the macaque hippocampus. J Neurophysiol 98:898-910.

Smith ME, Halgren E (1989) Dissociation of recognition memory components following temporal lobe lesions. J Exp Psychol Learn Mem Cogn 15:50-60.

Staresina BP, Bergmann TO, Bonnefond M, van der Meij R, Jensen O, Deuker L, Elger CE, Axmacher N, Fell J (2015) Hierarchical nesting of slow oscillations, spindles and ripples in the human hippocampus during sleep. Nat Neurosci 18:1679-1686.

Steriade M, Timofeev I (2003) Neuronal plasticity in thalamocortical networks during sleep and waking oscillations. Neuron 37:563-576.

Strange BA, Witter MP, Lein ES, Moser EI (2014) Functional organization of the hippocampal longitudinal axis. Nat Rev Neurosci 15:655-669.

Tort AB, Komorowski R, Eichenbaum H, Kopell N (2010) Measuring phase-amplitude coupling between neuronal oscillations of different frequencies. J Neurophysiol 104:1195-1210.

Tulving E, Markowitsch HJ (1998) Episodic and declarative memory: role of the hippocampus. Hippocampus 8:198-204.

Vargha-Khadem F, Gadian DG, Watkins KE, Connelly A, Van Paesschen W, Mishkin M (1997) Differential effects of early hippocampal pathology on episodic and semantic memory. Science 277:376-380.

Voytek B, D’Esposito M, Crone N, Knight RT (2013) A method for eventrelated phase/amplitude coupling. Neuroimage 64:416-424.

West MJ, Gundersen HJ (1990) Unbiased stereological estimation of the number of neurons in the human hippocampus. J Comp Neurol 296:1-22.

West MJ, Slomianka L, Gundersen HJ (1991) Unbiased stereological estimation of the total number of neurons in the subdivisions of the rat hippocampus using the optical fractionator. Anat Rec 231:482-497.

Wilson MA, McNaughton BL (1994) Reactivation of hippocampal ensemble memories during sleep. Science 265:676-679. 